\title{
The Theory and Application of Upwind Finite Difference Fractional Steps Procedure for Seawater Intrusion
}

\author{
Yirang Yuan, Hongxing Rui, Dong Liang, Changfeng Li \\ Institute of Mathematics, Shandong University, Jinan, China \\ Email: yryuan@sdu.edu.cn
}

Received August 14, 2012; revised September 10, 2012; accepted October 12, 2012

\begin{abstract}
Numerical simulation and theoretical analysis of seawater intrusion is the mathematical basis for modern environmental science. Its mathematical model is the nonlinear coupled system of partial differential equations with initial-boundary problems. For a generic case of a three-dimensional bounded region, two kinds of finite difference fractional steps procedures are put forward. Optimal order estimates in $l^{2}$ norm are derived for the error in the approximation solution. The present method has been successfully used in predicting the consequences of seawater intrusion and protection projects.
\end{abstract}

Keywords: Seawater Intrusion; Three-Dimensional Region; Upwind Fractional Steps; $l^{2} \quad$ Norm Estimate; Numerical Simulation

\section{Introduction}

Seawater intrusion refers to the invasion of salt water into the groundwater in coastal areas caused by the changes in natural water environment and social and economic development. In recent years, it has occurred in many countries in the world such as USA, Netherlands, Israel and Japan. After 1970s, the northern coastal area of our country, especially economic zones around Bohai such as Shandong, Hebei and Liaoning, is getting more and more seriously affected by this problem with Shandong province standing out. It leads to the great decrease in industrial and agricultural production, making people's living conditions, especially their drinking water, poorer and poorer. Therefore, it is urgent that seawater intrusion be completely tackled.

The mathematical model consists of water head equation and salt concentration equation. Because of the compressibility of porous media and that of the fluid, the change in fluid density with the salt concentration, and with the consideration of the fact that the salt is in the moving state in porous media, there may occur convection-dominated diffusion. While water is moving in the water-bearing stratum, it carries salt. The movement of this solute with underground water is called solute convection. Since salt is inhomogeniously distributed in the whole solution, it always diffuses from places with higher concentration to places with lower concentration even if the solution does not move.

\subsection{Water Head Equation}

With Darcy's law, Euler method and Huyakorn's linearization method, the water head equation is obtained $[1,2]$.

$$
\begin{aligned}
& S_{s} \frac{\partial H}{\partial t}-\nabla \cdot\left(K\left(\nabla H-\eta c \boldsymbol{e}_{3}\right)\right) \\
& =-\psi \eta \frac{\partial c}{\partial t}+\frac{\rho}{\rho_{0}} q,(x, y, z)^{T} \in \Omega, t \in J=(0, T],
\end{aligned}
$$

where $S_{s}$ is the specific retention, $H=p /\left(\rho_{0} g\right)-z$ is water head function, $p$ stands for pressure, $\rho_{0}$ represents the density of reference water (fresh water), $g$ is gravitational acceleration, $z$ is the height of water containing layer, $\rho$ is density and depends only on the concentration of salt $c$, Hugakorn's linearization $\rho=\rho_{0}\left(1+\varepsilon c / c_{s}\right)$ is adopted. $c_{s}$ is the concentration corresponding to the maximum density, and $\varepsilon$ is the density difference ration $\varepsilon=\left(\rho_{s}-\rho_{0}\right) / \rho_{0} . K=K \rho g / \mu$, $\mu$ is viscosity of the fluid, $\eta=\varepsilon / c_{s}$ is the density coupling coefficient. $\boldsymbol{e}_{3}=(0,0,1)^{\mathrm{T}}, \psi$ is the porosity, $q$ is source or sink term, and the permeability is noted by

$$
K=\left[\begin{array}{ccc}
K_{1} & 0 & 0 \\
0 & K_{2} & 0 \\
0 & 0 & K_{3}
\end{array}\right] .
$$

\subsection{Salt Concentration Equation}

The movement of $\mathrm{Cl}^{-}$dissolved in the fluid causes 
convection and diffusion of $\mathrm{Cl}^{-}$in porous media. From Fick's law and mass conservation law we have the following concentration Equation $[1,2]$.

$$
\begin{aligned}
& \psi \frac{\rho_{0}}{\rho} \frac{\partial c}{\partial t}=\nabla \cdot(\psi D \nabla c)-\boldsymbol{u} \cdot \nabla c \\
& +q\left(C^{*}-c\right),(x, y, z)^{\mathrm{T}} \in \Omega, t \in J,
\end{aligned}
$$

where $c$ stands for the concentration of $\mathrm{Cl}^{-}, C^{*}$ is the salt concentration near the source well. Darcy's velocity and the diffusion matrix are denoted by

$$
\boldsymbol{u}=-\frac{\rho_{0}}{\rho} K\left(\nabla H-\eta c \boldsymbol{e}_{3}\right)
$$

and

$$
D=\left[\begin{array}{lll}
D_{11} & D_{12} & D_{13} \\
D_{21} & D_{22} & D_{23} \\
D_{31} & D_{32} & D_{33}
\end{array}\right]
$$

\subsection{Initial Boundary Value Conditions}

To make a complete system, appropriate initial boundary value conditions are necessary in addition to the above equations. The initial value condition is

$$
\begin{aligned}
H(x, y, z, 0) & =H_{0}(x, y, z), c(x, y, z, 0) \\
& =c_{0}(x, y, z),(x, y, z) \in \Omega .
\end{aligned}
$$

There are three kinds of boundary value conditions. When concentration and water head are known, the first kind of boundary value condition can be given as

$$
\begin{aligned}
H(x, y, z, t) & =h(x, y, z, t), c(x, y, z, t) \\
& =g(x, y, z, t),(x, y, z) \in \Gamma_{1}, t \in J .
\end{aligned}
$$

The second kind of boundary value condition can be given to non-flow boundary:

$$
\boldsymbol{u} \cdot \boldsymbol{n}=0, D \nabla c \cdot \boldsymbol{n}=0,(x, y, z) \in \Gamma_{2}, t \in J
$$

where $\boldsymbol{n}$ is the unit vector in outer normal direction. A kind of Stefan boundary condition is for free surface boundary.

The boundary condition of water heat equation:

$$
\begin{array}{cc}
H=-z, & (x, y, z) \in \Gamma_{3} \\
\frac{\partial H}{\partial t}+\frac{1}{\mu}(\boldsymbol{u}-\boldsymbol{w}) \cdot \nabla(H+z)=0, & (x, y, z) \in \Gamma_{3}, t \in J .
\end{array}
$$

The boundary condition of salt concentration equation:

$$
\begin{aligned}
\psi D \nabla c \cdot \boldsymbol{n} & =\left(1-\mu_{0}\right)(\boldsymbol{u}-\boldsymbol{w}) \cdot \boldsymbol{n}\left(c-c^{\prime}\right), \\
(x, y, z) & \in \Gamma_{3}, t \in J .
\end{aligned}
$$

where $\boldsymbol{w}$ is the permeated fluid flow rate in a unit area and $c^{\prime}$ is the concentration of $\mathrm{Cl}^{-}$in permeated fluid.

In the study of seawater intrusion miscible model, for the miscible fluid Henry suggested an analytic solution under the simplified boundary condition and with the steady-state flow in the homogeneous medium [3]. Segol, Pinder, Grug, Heinrich and others studied the twodimensional cut plane problem [4,5], and Huyakorn, Gupta and Yapa studied the solving process of the threedimensional problem $[5,6]$. However, their calculations are made in specifically assumed conditions; therefore, they can not truly reflect seawater intrusion.

For plane incompressible two-phase displacement which is assumed to be $\Omega$-periodic, Jim Douglas, Ewing, Russell, and others have published famous papers on the characteristic finite difference method and finite element method to solve the convection-dominated diffusion problems with finite difference method, and to overcome oscillation and faults likely to occur in the traditional methods [7-12]. For compressible two-phase displacement problem, Douglas and others have contributed much to the mathematical model of "infinitesimal compressibility", numerical method and analysis [13-16]. Douglas and Yuan discarded periodic conditions, put forward a new modified characteristic finite difference method and finite element method, and obtained optimal order estimation in $l^{2}$ norm [17-20]. Special treatment is needed for characteristic lines because the method of characteristics asks for interpolation and they may go through the boundaries near the solution regions. It is necessary to find out the intersection point of characteristic lines and mesh boundaries and calculate their corresponding functional values. While such calculation is designed, we must determine whether characteristic lines really go through the boundaries in order to decide whether time steps lengths should be changed. In a word, the practical calculation is quite complicated $[19,20]$.

For the convection-dominated diffusion problems, Axelsson, Ewing, Lazarov and others proposed upwind finite difference method [21-23] to overcome oscillation and to avoid computation complexity of the characteristic differential method near boundary meshes. Douglas and Peaceman applied the alternating-direction method to numerical reservoir simulation [24,25]. By using Fourier analysis, they succeeded in proving the stability and convergence according to the constant coefficient [26,27]. This paper, starting from the actual case, puts forward the modified method of upwind with finite difference fractional steps procedure for seawater intrusion. It can overcome oscillation and diffusion and computative complexity. At the same time it can convert a three-dimensional problem into three successive one-dimensional problems, reducing computation complexity and making computation practical. Moreover, it increases the space calculation accuracy to the second order. Some techniques, such as calculus of variations, energy method, operator-splitting, upwind method, commutative law of 
multiplication of difference operators, decomposition of high order difference operators, the theory of prior estimates and techniques are adopted. Optimal order estimates in $l^{2}$ norm are derived to determine the error in the approximate solution. Thus the difficult problem has been solved $[16,28]$.

Generally, this is a positive definite problem:

$$
\begin{aligned}
& 0<K_{*} \leq K_{i}(c) \leq K^{*},\left|\partial^{2} K_{i}(c) / \partial t^{2}\right| \leq K^{*}, i=1,2,3, \\
& 0<D_{*} \leq D(x, y, z, t) \leq D^{*}, 0<\Psi_{*} \leq \Psi(x, y, z) \leq \Psi^{*},
\end{aligned}
$$

where $K_{*}, K^{*}, \Psi_{*}, \Psi^{*}$ are constants.

Our assumptions on the regularity of the solution of (1)-(5) are denoted collectively by

$$
\begin{aligned}
& H, c \in L^{\infty}\left(W^{4, \infty}\right) \cap W^{1, \infty}\left(W^{1, \infty}\right), \\
& \partial^{2} H / \partial t^{2}, \partial^{2} c / \partial t^{2} \in L^{\infty}\left(L^{\infty}\right) .
\end{aligned}
$$

In this paper $M$ and $\varepsilon$ express general positive constant and general positive small constant respectively, and have different meanings.

\section{The Upwind Finite Difference Fractional Steps Procedure}

For brevity we consider only the first kind of boundary value problem and the diffusion matrix $D(x, y, z)$ of diagonal form. In order to get the solution by using finite difference method we use mesh region $\Omega_{h}$ instead of region $\Omega$. On space $(x, y, z)$, let step lengths be $h_{3}, x_{\mathrm{i}}=$ $i h_{1}, y_{\mathrm{j}}=j h_{2}, z_{\mathrm{k}}=k h_{3}$,

$$
\Omega_{h}=\left\{\left(x_{i}, y_{j}, z_{k}\right) \mid \begin{array}{l}
i_{1}(j, k)<i<i_{2}(j, k) \\
j_{1}(i, k)<j<j_{2}(i, k) \\
k_{1}(i, j)<k<k_{2}(i, j)
\end{array}\right\} .
$$

Let $\partial \Omega_{h}$ stands for the boundaries of $\Omega_{h}, X_{i j k}=\left(i h_{1}\right.$, $\left.j h_{2}, k h_{3}\right)^{\mathrm{T}}, t^{n}=n \Delta t, W\left(X_{i j k}, t^{n}\right)=W_{i j k}^{n}$. Let

$$
\begin{aligned}
& K\left(C_{h}^{n}\right)_{i+1 / 2, j k}=\left[K\left(X_{i j k}, C_{h, j k}^{n}\right)+K\left(X_{i+1, j k}, C_{h, i+1, j k}^{n}\right)\right] / 2, \\
& K\left(C_{h}^{n}\right)_{i, j+1 / 2, k}=\left[K\left(X_{i j k}, C_{h, i j k}^{n}\right)+K\left(X_{i, j+1, k}, C_{h, i, j+1, k}^{n}\right)\right] / 2, \\
& K\left(C_{h}^{n}\right)_{i j, k+1 / 2}=\left[K\left(X_{i j k}, C_{h, j i k}^{n}\right)+K\left(X_{i j, k+1}, C_{h, i j, k+1}^{n}\right)\right] / 2,
\end{aligned}
$$

Let $H_{h, i j k}^{n}$ and $C_{h, i j k}^{n}$ be the finite difference solutions of $H\left(X_{i j k}, t^{n}\right)$ and $c\left(X_{i j k}, t^{n}\right)$, respectively. If the finite difference solutions $C_{h}^{n}$ and $H_{h}^{n}$ are known, we find the finite difference solutions $C_{h}^{n+1}, H_{h}^{n+1}$ at $t^{n+1}$.

\subsection{The Second Order Upwind Finite Difference Fractional Steps Scheme}

First, compute the approximate Darcy's velocity $U^{n}=\left(U_{1}^{n}, U_{2}^{n}, U_{3}^{n}\right)^{T}$ as follows:

$$
\begin{gathered}
U_{1}^{n}=-\frac{\rho_{0}}{2}\left\{\left[\frac{K_{1}\left(C_{h}^{n}\right)}{\rho\left(C_{h}^{n}\right)}\right]_{i+1 / 2, j k} \frac{H_{h, i+1, j k}^{n}-H_{h, j k}^{n}}{h_{1}}+\left[\frac{K_{1}\left(C_{h}^{n}\right)}{\rho\left(C_{h}^{n}\right)}\right]_{i-1 / 2, j k} \frac{H_{h, j k}^{n}-H_{h, i-1, j k}^{n}}{h_{1}}\right\}, \\
U_{2}^{n}=-\frac{\rho_{0}}{2}\left\{\left[\frac{K_{2}\left(C_{h}^{n}\right)}{\rho\left(C_{h}^{n}\right)}\right]_{i, j+1 / 2, k} \frac{H_{h, i, j+1, k}^{n}-H_{h, j k}^{n}}{h_{2}}+\left[\frac{K_{2}\left(C_{h}^{n}\right)}{\rho\left(C_{h}^{n}\right)}\right]_{i, j-1 / 2, k} \frac{H_{h, i j k}^{n}-H_{h, i, j-1, k}^{n}}{h_{2}}\right\}, \\
U_{3}^{n}=-\frac{\rho_{0}}{2}\left\{\left[\frac{K_{3}\left(C_{h}^{n}\right)}{\rho\left(C_{h}^{n}\right)}\right]_{i j, k+1 / 2} \frac{H_{h, i j, k+1}^{n}-H_{h, i j k}^{n}}{h_{3}}+\left[\frac{K_{3}\left(C_{h}^{n}\right)}{\rho\left(C_{h}^{n}\right)}\right]_{i j, k-1 / 2} \frac{H_{h, i j k}^{n}-H_{h, i j, k-1}^{n}}{h_{3}}\right\} .
\end{gathered}
$$

For salt concentration Equation (2), the modified method of upwind with finite difference fractional steps 
scheme is given by

$$
\begin{aligned}
& \beta\left(C_{h, i j k}^{n}\right) \frac{C_{h, j k}^{n+1 / 3}-C_{h, j k}^{n}}{\Delta t} \\
& =\left(1+\frac{h_{1}}{2}\left|U_{1}^{n}\right| \bar{D}_{1}^{-1}\right)_{i j k}^{-1} \delta_{\bar{x}}\left(\bar{D}_{1} \delta_{x} C_{h}^{n+1 / 3}\right)_{i j k} \\
& +\left(1+\frac{h_{2}}{2}\left|U_{2}^{n}\right| \bar{D}_{2}^{-1}\right)_{i j k}^{-1} \delta_{\bar{y}}\left(\bar{D}_{2} \delta_{y} C_{h}^{n}\right)_{i j k} \\
& +\left(1+\frac{h_{3}}{2}\left|U_{3}^{n}\right| \bar{D}_{3}^{-1}\right)_{i j k}^{-1} \delta_{\bar{Z}}\left(\bar{D}_{3} \delta_{z} C_{h}^{n}\right)_{i j k} \\
& -\delta_{U_{1}^{n}, x} C_{h, i j k}^{n}-\delta_{U_{2}^{n}, y} C_{h, i j k}^{n}-\delta_{U_{3}^{n}, z} C_{h, i j k}^{n} \\
& +q_{i j k}^{n}\left(C_{h, i j k}^{*, n}-C_{h, i j k}^{n}\right), i_{1}(j, k)<i<i_{2}(j, k), \\
& C_{h, j i k}^{n+1 / 3}=g_{i j k}^{n+1}, X_{i j k} \in \partial \Omega_{h},
\end{aligned}
$$

where $\beta(C)=\psi \rho_{0} / \rho, \bar{D}_{i}=\psi D_{i}, i=1,2,3$.

$$
\begin{aligned}
& \beta\left(C_{h, i j k}^{n}\right) \frac{C_{h, j k}^{n+2 / 3}-C_{h, j i k}^{n+1 / 3}}{\Delta t} \\
& =\left(1+\frac{h_{2}}{2}\left|U_{2}^{n}\right| \bar{D}_{2}^{-1}\right)_{i j k}^{-1} \delta_{\bar{y}}\left(\bar{D}_{2} \delta_{y}\left(C_{h}^{n+2 / 3}-C_{h}^{n}\right)\right)_{i j k}, \\
& j_{1}(i, k)<j<j_{2}(i, k), \\
& C_{h, j i k}^{n+2 / 3}=g_{i j k}^{n+1}, X_{i j k} \in \partial \Omega_{h}, \\
& \beta\left(C_{h, i j k}^{n}\right) \frac{C_{h, j i k}^{n+1}-C_{h, i j k}^{n+2 / 3}}{\Delta t} \\
& =\left(1+\frac{h_{3}}{2}\left|U_{3}^{n}\right| \bar{D}_{3}^{-1}\right)_{i j k}^{-1} \delta_{\bar{z}}\left(\bar{D}_{3} \delta_{z}\left(C_{h}^{n+1}-C_{h}^{n}\right)\right)_{i j k} \\
& k_{1}(i, j)<k<k_{2}(i, j), \\
& C_{h, i j k}^{n+1}=g_{i j k}^{n+1}, X_{i j k} \in \partial \Omega_{h},
\end{aligned}
$$

where

$$
\begin{aligned}
& \delta_{U_{1}^{n}, x} c_{i j k}=U_{1, i j k}^{n}\left[\bar{H}\left(U_{1, j k}^{n}\right) \bar{D}_{1, i j k}^{-1} \cdot \bar{D}_{1, i-1 / 2, j k} \delta_{\bar{x}}+\left(1-\bar{H}\left(U_{1, j k}^{n}\right)\right) \bar{D}_{1, i j k}^{-1} \cdot \bar{D}_{1, i+1 / 2, j k} \delta_{x}\right] c_{i j k}, \\
& \delta_{U_{2}^{n}, y} c_{i j k}=U_{2, j k k}^{n}\left[\bar{H}\left(U_{2, i j k}^{n}\right) \bar{D}_{2, i j k}^{-1} \cdot \bar{D}_{2, i, j-1 / 2, k} \delta_{\bar{y}}+\left(1-\bar{H}\left(U_{2, j k k}^{n}\right)\right) \bar{D}_{2, i j k}^{-1} \cdot \bar{D}_{2, i, j+1 / 2, k} \delta_{y}\right] c_{i j k}, \\
& \delta_{U_{3}^{n}, 2} c_{i j k}=U_{3, i j k}^{n}\left[\bar{H}\left(U_{3, i j k}^{n}\right) \bar{D}_{3, i j k}^{-1} \cdot \bar{D}_{3, i j, k-1 / 2} \delta_{\bar{z}}+\left(1-\bar{H}\left(U_{3, i j k}^{n}\right)\right) \bar{D}_{3, i j k}^{-1} \cdot \bar{D}_{3, i j, k+1 / 2} \delta_{z}\right] c_{i j k},
\end{aligned}
$$

and $\bar{H}(z)=\left\{\begin{array}{l}1, z \geq 0 \\ 0, z<0\end{array}\right.$.

Next, for fluid Equation (1) the fractional steps sinite difference scheme is given by

$$
\begin{aligned}
& S_{s, i j k} \frac{H_{h, i j k}^{n+1 / 3}-H_{h, i j k}^{n}}{\Delta t}=\delta_{\bar{x}}\left(K_{1}\left(C_{h}^{n}\right) \delta_{x} H_{h}^{n+1 / 3}\right)_{i j k} \\
& +\delta_{\bar{y}}\left(K_{2}\left(C_{h}^{n}\right) \delta_{y} H_{h}^{n}\right)_{i j k}+\delta_{\bar{z}}\left(K_{3}\left(C_{h}^{n}\right) \delta_{z} H_{h}^{n}\right)_{i j k} \\
& -\eta \psi_{i j k} \frac{C_{h, j k}^{n+1}-C_{h, i j k}^{n}}{\Delta t}+\frac{\rho\left(C_{h, i j k}^{n}\right)}{\rho_{0}} q_{i j k}^{n} \\
& +\eta \delta_{\bar{z}}\left(K_{3}\left(C_{h}^{n}\right) C_{h}^{n}\right)_{i j k}, i_{1}(j, k)<i<i_{2}(j, k), \\
& H_{h, i j k}^{n+1 / 3}=h_{i j k}^{n+1}, X_{i j k} \in \partial \Omega_{h}, \\
& S_{s, i j k} \frac{H_{h, i j k}^{n+2 / 3}-H_{h, j j k}^{n+1 / 3}}{\Delta t} \\
& =\delta_{\bar{y}}\left(K_{2}\left(C_{h}^{n}\right) \delta_{y}\left(H_{h}^{n+2 / 3}-H_{h}^{n}\right)\right)_{i j k}, \\
& j_{1}(i, k)<j<j_{2}(i, k), \\
& H_{h, j i k}^{n+2 / 3}=h_{i j k}^{n+1}, X_{i j k} \in \partial \Omega_{h},
\end{aligned}
$$

$$
\begin{aligned}
& S_{s, i j k} \frac{H_{h, i j k}^{n+1}-H_{h, j k k}^{n+2 / 3}}{\Delta t} \\
& =\delta_{\bar{z}}\left(K_{3}\left(C_{h}^{n}\right) \delta_{z}\left(H_{h}^{n+1}-H_{h}^{n}\right)\right)_{i j k}, \\
& k_{1}(i, j)<k<k_{2}(i, j), \\
& H_{h, j i k}^{n+1}=h_{i j k}^{n+1}, X_{i j k} \in \partial \Omega_{h} .
\end{aligned}
$$

The initial approximation reads

$$
C_{h, i j k}^{0}=c_{0}\left(X_{i j k}\right), H_{h, i j k}^{0}=H_{0}\left(X_{i j k}\right), X_{i j k} \in \Omega_{h} .
$$

The algorithm for a time step is as follows: Assuming the Approximate solution $\left\{C_{h, j k}^{n}, H_{h, j k}^{n}\right\}$ at time $t^{n}$ is known, it is needed to find out the approximate solutions at the next time level. First, compute Darcy's velocity $\bar{U}^{n}$, from schemes (8a), (8b), and method of speedup is used to get the solution of transition sheaf $\left\{C_{h, i j k}^{n+1 / 3}\right\}$ along $x$ direction. Secondly, from schemes (9a), (9b) we obtain the solution of transition sheaf $\left\{C_{h, j k}^{n+2 / 3}\right\}$. Thirdly, from schemes (10a), (10b) we obtain solution $\left\{C_{h, i j k}^{n+1}\right\}$. Next, from (11a), (11b), by using method of speedup, we get the solution of transition sheaf $\left\{H_{h, j i k}^{n+1 / 3}\right\}$ along $x$ direction; from (12a), (12b) we obtain the solution of transition sheaf $\left\{H_{h, i j k}^{n+2 / 3}\right\}$. Finally, from schemes (13a), (13b) we obtain solution $\left\{H_{h, i j k}^{n+1}\right\}$. So a complete time 
step can be taken. At last, it is because of the positive condition that this finite difference solution exists, being the sole one.

\subsection{The First Order Weighted Upwind Finite Difference Fractional Steps Scheme}

For salt concentration Equation (2), the first order upwind finite difference fractional steps scheme is given by

$$
\begin{aligned}
& \beta\left(C_{h, i j k}^{n}\right) \frac{C_{h, j k}^{n+1 / 3}-C_{h, i j k}^{n}}{\Delta t}=\delta_{\bar{x}}\left(\bar{D}_{1} \delta_{x} C_{h}^{n+1 / 3}\right)_{i j k} \\
& +\delta_{\bar{y}}\left(\bar{D}_{2} \delta_{y} C_{h}^{n}\right)_{i j k}+\delta_{\bar{z}}\left(\bar{D}_{3} \delta_{z} C_{h}^{n}\right)_{i j k} \\
& -\bar{\delta}_{U_{1}^{n}, x} C_{h, j i k}^{n}-\bar{\delta}_{U_{2}^{n}, y} C_{h, j i k}^{n}-\bar{\delta}_{U_{3}^{n}, z} C_{h, j i k}^{n} \\
& +q_{i j k}^{n}\left(C_{h, i j k}^{*, n}-C_{h, i j k}^{n}\right), i_{1}(j, k)<i<i_{2}(j, k), \\
& C_{h, i j k}^{n+1 / 3}=g_{i j k}^{n+1}, X_{i j k} \in \partial \Omega_{h}, \\
& \beta\left(C_{h, j k k}^{n}\right) \frac{C_{h, j i k}^{n+2 / 3}-C_{h, i j k}^{n+1 / 3}}{\Delta t}=\delta_{\bar{y}}\left(\bar{D}_{2} \delta_{y}\left(C_{h}^{n+2 / 3}-C_{h}^{n}\right)\right)_{i j k} \text {, } \\
& j_{1}(i, k)<j<j_{2}(i, k) \text {, } \\
& C_{h, j k}^{n+2 / 3}=g_{i j k}^{n+1}, X_{i j k} \in \partial \Omega_{h}, \\
& \beta\left(C_{h, j k}^{n}\right) \frac{C_{h, j k}^{n+1}-C_{h, j k}^{n+2 / 3}}{\Delta t}=\delta_{\bar{z}}\left(\bar{D}_{3} \delta_{z}\left(C_{h}^{n+1}-C_{h}^{n}\right)\right)_{i j k}, \\
& k_{1}(i, j)<k<k_{2}(i, j) \text {, } \\
& C_{h, j i k}^{n+1}=g_{i j k}^{n+1}, X_{i j k} \in \partial \Omega_{h},
\end{aligned}
$$

where

$$
\begin{aligned}
\bar{\delta}_{U_{1}^{n}, x} c_{i j k} & =\alpha U_{1, j i k}^{n}\left[\bar{H}\left(U_{1, j k}^{n}\right) \delta_{\bar{x}}+\left(1-\bar{H}\left(U_{1, j k}^{n}\right)\right) \delta_{x}\right] c_{i j k} \\
& +(1-\alpha) U_{1, j k}^{n}\left(c_{i+1, j k}-c_{i-1, j k}\right) / 2 h_{1},
\end{aligned}
$$

and $\bar{\delta}_{U^{n}, y} C_{i j k}, \bar{\delta}_{U_{3}^{n}, z} c_{i j k}$ are defined similarly with a constant $\alpha \in[0,1]$.

The algorithm is similar to that of Scheme (8)-(13).

\section{Convergence Analysis}

For brevity we assume $\Omega=\{[0,1]\}^{3}, h=1 / N$, $X_{i j k}=(i h, j h, k h)^{\mathrm{T}}, \quad t^{n}=n \Delta t, \quad W\left(X_{i j k}, t^{n}\right)=W_{i j k}^{n}$. Let $\pi=H-H_{h}, \xi=c-C_{h}$, where $H$ and $c$ are the exact solutions of this problem (1) - (5), and $H_{h}$ and $C_{h}$ are the difference solutions of the schemes (8) - (13). $\langle\cdot, \cdot\rangle,\|\cdot\|$ and \|\|$_{1}$ denote the inner product and the norms on the discrete spaces $l^{2}(\Omega)$ and $h^{1}(\Omega)$ corresponding to $L^{2}(\Omega)$ and $H^{1}(\Omega)[19,20,29]$. First consider the second order scheme.

Theorem I. Suppose that the exact solutions of problem (1)-(5) satisfy condition:

$$
\begin{aligned}
& H, c \in W^{1, \infty}\left(W^{1, \infty}\right) \cap L^{\infty}\left(W^{4, \infty}\right), \\
& \partial H / \partial t, \partial c / \partial t \in L^{\infty}\left(W^{4, \infty}\right), \partial^{2} H / \partial t^{2}, \partial^{2} c / \partial t^{2} \in L^{\infty}\left(L^{\infty}\right) .
\end{aligned}
$$

Adopt the modified method of upwind procedures (8)(13). Let the dissectible satisfy relation: $\Delta t=O\left(h^{2}\right)$. Then the following error estimates hold:

$$
\begin{aligned}
& \left\|H-H_{h}\right\|_{\bar{L}^{\infty}\left(J ; h^{1}\right)}+\left\|c-C_{h}\right\|_{\bar{L}^{\infty}\left(J ; h^{1}\right)}+\left\|d_{t}\left(H-H_{h}\right)\right\|_{\bar{L}^{2}\left(J ; l^{2}\right)} \\
& +\left\|d_{t}\left(c-C_{h}\right)\right\|_{\bar{L}^{2}\left(J ; l^{2}\right)} \leq M^{*}\left(\Delta t+h^{2}\right)
\end{aligned}
$$

where $\|g\|_{\bar{L}^{\infty}(J ; S)}=\sup _{n \Delta t \leq T}\left\|g^{n}\right\|_{S},\|g\|_{\bar{L}^{2}(J ; S)} \sup _{N \Delta t \leq T}\left\{\sum_{n=0}^{N}\left\|g^{n}\right\|_{S}^{2} \Delta t\right\}$.

Constant $M^{*}$ depends on $H, C$ and their derivatives.

Proof. First consider the concentration equation. For Equations (8)-(13), dispel $C_{h}^{n+1 / 3}$ and $C_{h}^{n+2 / 3}$, and we get the following equivalent form:

$$
\begin{aligned}
& \beta\left(C_{h, i j k}^{n}\right) \frac{C_{h, j i k}^{n+1}-C_{h, j k}^{n}}{\Delta t}-\left\{\left(1+\frac{h}{2}\left|U_{1}^{n}\right| \bar{D}_{1}^{-1}\right)_{i j k}^{-1} \delta_{\bar{x}}\left(\bar{D}_{1} \delta_{x} C_{h}^{n+1}\right)_{i j k}\right. \\
& +\left(1+\frac{h}{2}\left|U_{2}^{n}\right| \bar{D}_{2}^{-1}\right)_{i j k}^{-1} \delta_{\bar{y}}\left(\bar{D}_{2} \delta_{y} C_{h}^{n+1}\right)_{i j k} \\
& \left.+\left(1+\frac{h}{2}\left|U_{3}^{n}\right| \bar{D}_{3}^{-1}\right)_{i j k}^{-1} \delta_{\bar{Z}}\left(\bar{D}_{3} \delta_{z} C_{h}^{n+1}\right)_{i j k}\right\} \\
& =-\delta_{U_{1}^{n}, x} C_{h, i j k}^{n}-\delta_{U_{2}^{n}, y} C_{h, j i k}^{n}-\delta_{U_{3}^{n}, z} C_{h, i j k}^{n}+q_{i j k}^{n}\left(C_{h, i j k}^{*, n}-C_{h, i j k}^{n}\right) \\
& -(\Delta t)^{2}\left\{( 1 + \frac { h } { 2 } | U _ { 1 } ^ { n } | \overline { D } _ { 1 } ^ { - 1 } ) _ { i j k } ^ { - 1 } \delta _ { \overline { x } } \left(\overline { D } _ { 1 } \delta _ { x } \left(\beta^{-1}\left(C_{h}^{n}\right)\right.\right.\right. \\
& \left.\left.\cdot\left(1+\frac{h}{2}\left|U_{2}^{n}\right| \bar{D}_{2}^{-1}\right)^{-1} \delta_{\bar{y}}\left(\bar{D}_{2} \delta_{y} d_{t} C_{h}^{n}\right)\right)\right)_{i j k} \\
& +\left(1+\frac{h}{2}\left|U_{1}^{n}\right| \bar{D}_{1}^{-1}\right)^{-1} \delta_{\bar{x} k}\left(\overline { D } _ { 1 } \delta _ { x } \left(\beta^{-1}\left(C_{h}^{n}\right)\right.\right. \\
& \left.\left.\cdot\left(1+\frac{h}{2}\left|U_{3}^{n}\right| \bar{D}_{3}^{-1}\right)^{-1} \delta_{\bar{z}}\left(\bar{D}_{3} \delta_{z} d_{t} C_{h}^{n}\right)\right)\right)_{i j k} \\
& +\left(1+\frac{h}{2}\left|U_{2}^{n}\right| \bar{D}_{2}^{-1}\right)_{i j k}^{-1} \delta_{\bar{y}}\left(\overline { D } _ { 1 } \delta _ { y } \left(\beta^{-1}\left(C_{h}^{n}\right)\right.\right. \\
& \left.\left.\left.\cdot\left(1+\frac{h}{2}\left|U_{3}^{n}\right| \bar{D}_{3}^{-1}\right)^{-1} \delta_{\bar{z}}\left(\bar{D}_{3} \delta_{z} d_{t} C_{h}^{n}\right)\right)\right)_{i j k}\right\} \\
& +(\Delta t)^{3}\left(1+\frac{h}{2}\left|U_{1}^{n}\right| \bar{D}_{1}^{-1}\right)_{i j k}^{-1} \delta_{\bar{x}} \\
& \cdot\left(\overline { D } _ { 1 } \delta _ { x } \left(\beta ^ { - 1 } ( C _ { h } ^ { n } ) ( 1 + \frac { h } { 2 } | U _ { 2 } ^ { n } | \overline { D } _ { 2 } ^ { - 1 } ) ^ { - 1 } \delta _ { \overline { y } } \left(\bar{D}_{2} \delta_{y}\right.\right.\right. \\
& \left.\left.\left.\left.\beta^{-1}\left(C_{h}^{n}\right)\left(1+\frac{h}{2}\left|U_{3}^{n}\right| \bar{D}_{3}^{-1}\right)^{-1} \delta_{\bar{z}}\left(\bar{D}_{3} \delta_{z} d_{t} C_{h}^{n}\right)\right)\right)\right)\right)_{i j k},
\end{aligned}
$$$$
1 \leq i, j, k \leq N-1,
$$ 


$$
C_{h, i j k}^{n+1}=g_{i j k}^{n+1}, X_{i j k} \in \partial \Omega_{h}
$$

From Equation (2) $\left(t=t^{n+1}\right)$ and (16) we have the concentration error equations.

$$
\begin{aligned}
& \beta\left(C_{h, i j k}^{n}\right) \frac{\xi_{h, i j k}^{n+1}-\xi_{h, i j k}^{n}}{\Delta t}-\left\{\left(1+\frac{h}{2}\left|U_{1}^{n}\right| \bar{D}_{1}^{-1}\right)^{-1} \delta_{\bar{x}}\left(\bar{D}_{1} \delta_{x} \xi_{h}^{n+1}\right)_{i j k}\right. \\
& \left.+\left(1+\frac{h}{2}\left|U_{2}^{n}\right| \bar{D}_{2}^{-1}\right)_{i j k}^{-1} \delta_{\bar{y}}\left(\bar{D}_{2} \delta_{y} \xi_{h}^{n+1}\right)_{i j k}+\left(1+\frac{h}{2}\left|U_{3}^{n}\right| \bar{D}_{3}^{-1}\right)_{i j k}^{-1} \delta_{\bar{z}}\left(\bar{D}_{3} \delta_{z} \xi_{h}^{n+1}\right)_{i j k}\right\} \\
& =\left[\delta_{U_{1}^{n}, X} C_{h, i j k}^{n}-\delta_{u_{1}^{n+1}, x} C_{i j k}^{n+1}\right]+\left[\delta_{U_{2}^{n}, y} C_{h, i j k}^{n}-\delta_{u_{2}^{n+1}, y} C_{i j k}^{n+1}\right]+\left[\delta_{U_{3}^{n}, z} C_{h, i j k}^{n}-\delta_{u_{3}^{n+1}, z} C_{i j k}^{n+1}\right] \\
& +\left\{\left[\left(1+\frac{h}{2}\left|u_{1}^{n+1}\right| \bar{D}_{1}^{-1}\right)_{i j k}^{-1}-\left(1+\frac{h}{2}\left|U_{1}^{n}\right| \bar{D}_{1}^{-1}\right)_{i j k}^{-1}\right] \delta_{\bar{x}}\left(\bar{D}_{1} \delta_{x} C_{h}^{n+1}\right)_{i j k}\right. \\
& +\left[\left(1+\frac{h}{2}\left|u_{2}^{n+1}\right| \bar{D}_{2}^{-1}\right)_{i j k}^{-1}-\left(1+\frac{h}{2}\left|U_{2}^{n}\right| \bar{D}_{2}^{-1}\right)_{i j k}^{-1}\right] \delta_{\bar{y}}\left(\bar{D}_{2} \delta_{y} C_{h}^{n+1}\right)_{i j k} \\
& \left.+\left[\left(1+\frac{h}{2}\left|u_{3}^{n+1}\right| \bar{D}_{3}^{-1}\right)_{i j k}^{-1}-\left(1+\frac{h}{2}\left|U_{3}^{n}\right| \bar{D}_{3}^{-1}\right)_{i j k}^{-1}\right] \delta_{\bar{z}}\left(\bar{D}_{3} \delta_{z} C_{h}^{n+1}\right)_{i j k}\right\} \\
& +\left\{q_{i j k}^{n+1}\left(C_{h, i j k}^{*, n}-C_{i j k}^{n+1}\right)-q_{i j k}^{n}\left(C_{h, i j k}^{*, n}-C_{h, i j k}^{n}\right)\right\} \\
& -(\Delta t)^{2}\left(\left[\left(1+\frac{h}{2}\left|u_{1}^{n+1}\right| \bar{D}_{1}^{-1}\right)_{i j k}^{-1} \delta_{\bar{x}}\left(\bar{D}_{1} \delta_{x}\left(\beta^{-1}\left(c^{n+1}\right)\left(1+\frac{h}{2}\left|u_{2}^{n+1}\right| \bar{D}_{2}^{-1}\right)^{-1} \delta_{\bar{y}}\left(\bar{D}_{2} \delta_{y} d_{t} c^{n}\right)\right)\right)_{i j k}\right.\right. \\
& \left.-\left(1+\frac{h}{2}\left|U_{1}^{n}\right| \bar{D}_{1}^{-1}\right)_{i j k}^{-1} \delta_{\bar{x}}\left(\bar{D}_{1} \delta_{x}\left(\beta^{-1}\left(C_{h}^{n}\right)\left(1+\frac{h}{2}\left|U_{2}^{n}\right| \bar{D}_{2}^{-1}\right)^{-1} \delta_{\bar{y}}\left(\bar{D}_{2} \delta_{y} d_{t} C_{h}^{n}\right)\right)\right)_{i j k}\right] \\
& \left.+\left[\left(1+\frac{h}{2}\left|u_{1}^{n+1}\right| \bar{D}_{1}^{-1}\right)_{i j k}^{-1} \delta_{\bar{x}}\left(\bar{D}_{1} \delta_{x}\left(\beta^{-1}\left(c^{n+1}\right)\left(1+\frac{h}{2}\left|U_{2}^{n}\right| \bar{D}_{2}^{-1}\right)^{-1} \delta_{\bar{y}}\left(\bar{D}_{2} \delta_{y} d_{t} C_{h}^{n}\right)\right)\right)_{i j k}-\cdots\right]+\cdots+[\cdots]\right) \\
& +(\Delta t)^{3}\left\{( 1 + \frac { h } { 2 } | u _ { 1 } ^ { n + 1 } | \overline { D } _ { 1 } ^ { - 1 } ) _ { i j k } ^ { - 1 } \delta _ { \overline { x } } \left(\overline { D } _ { 1 } \delta _ { x } \left(\beta ^ { - 1 } ( c ^ { n + 1 } ) ( 1 + \frac { h } { 2 } | u _ { 2 } ^ { n + 1 } | \overline { D } _ { 2 } ^ { - 1 } ) ^ { - 1 } \delta _ { \overline { y } } \left(\bar{D}_{2} \delta_{y} \beta^{-1}\left(c^{n+1}\right)\right.\right.\right.\right. \\
& \left.\left.\left.\left.\cdot\left(1+\frac{h}{2}\left|u_{3}^{n+1}\right| \bar{D}_{3}^{-1}\right)^{-1} \delta_{\bar{z}}\left(\bar{D}_{3} \delta_{z} d_{t} c^{n}\right)\right)\right)\right)\right)_{i j k}-\left(1+\frac{h}{2}\left|U_{1}^{n}\right| \bar{D}_{1}^{-1}\right)_{i j k}^{-1} \delta_{\bar{x}} \\
& \cdot\left(\overline { D } _ { 1 } \delta _ { x } \left(\beta ^ { - 1 } ( C _ { h } ^ { n } ) ( 1 + \frac { h } { 2 } | U _ { 2 } ^ { n } | \overline { D } _ { 2 } ^ { - 1 } ) ^ { - 1 } \delta _ { \overline { y } } \left(\bar{D}_{2} \delta_{y} \beta^{-1}\left(C_{h}^{n}\right)\left(1+\frac{h}{2}\left|U_{3}^{n}\right| \bar{D}_{3}^{-1}\right)^{-1}\right.\right.\right. \\
& \left.\left.\left.\left.\left.\delta_{\bar{z}}\left(\bar{D}_{3} \delta_{z} d_{t} C_{h}^{n}\right)\right)\right)\right)\right)_{i j k}\right\}+\varepsilon_{1, j i k}^{n+1}, 1 \leq i, j, k \leq N-1,
\end{aligned}
$$$$
\xi_{i j k}^{n+1}=0, X_{i j k} \in \partial \Omega_{h},
$$

where $\left|\varepsilon_{1, j i k}^{n+1}\right| \leq M\left(\Delta t+h^{2}\right)$.

Next, consider the fluid equation. For Equations (11)-(13), dispel $H_{h}^{n+1 / 3}$ and $H_{h}^{n+2 / 3}$, and we get the following equivalent form: 


$$
\begin{aligned}
& S_{s, i j k} \frac{H_{h, j k \mathrm{k}}^{n+1}-H_{h, j \mathrm{k} k}^{n}}{\Delta t}-\left\{\delta_{\bar{x}}\left(K_{1}\left(C_{h}^{n}\right) \delta_{x} H_{h}^{n+1}\right)_{i j k}+\delta_{\bar{y}}\left(K_{2}\left(C_{h}^{n}\right) \delta_{y} H_{h}^{n+1}\right)_{i j k}+\delta_{\bar{z}}\left(K_{3}\left(C_{h}^{n}\right) \delta_{z} H_{h}^{n+1}\right)_{i j k}\right. \\
& =-\eta \psi_{i j k} \frac{C_{h, j i k}^{n+1}-C_{h, i j k}^{n}}{\Delta t}+\frac{\rho\left(C_{h, i j k}^{n}\right)}{\rho_{0}} q_{i j k}^{n}+\eta \delta_{\bar{z}}\left(K_{3}\left(C_{h}^{n}\right) C_{h}^{n}\right)_{i j k} \\
& -(\Delta t)^{2}\left\{\delta_{\bar{x}}\left(K_{1}\left(C_{h}^{n}\right) \delta_{x}\left(S_{s}^{-1} \delta_{\bar{y}}\left(K_{2}\left(C_{h}^{n}\right) \delta_{y} d_{t} H_{h}^{n}\right)\right)\right)_{i j k}\right. \\
& \left.+\delta_{\bar{x}}\left(K_{1}\left(C_{h}^{n}\right) \delta_{x}\left(S_{s}^{-1} \delta_{\bar{z}}\left(K_{3}\left(C_{h}^{n}\right) \delta_{z} d_{t} H_{h}^{n}\right)\right)\right)_{i j k}+\delta_{\bar{y}}\left(K_{2}\left(C_{h}^{n}\right) \delta_{y}\left(S_{s}^{-1} \delta_{\bar{z}}\left(K_{3}\left(C_{h}^{n}\right) \delta_{z} d_{t} H_{h}^{n}\right)\right)\right)_{i j k}\right\} \\
& \left.+(\Delta t)^{3} \delta_{\bar{x}}\left(K_{1}\left(C_{h}^{n}\right) \delta_{x}\left(S_{s}^{-1} \delta_{\bar{y}}\left(K_{2}\left(C_{h}^{n}\right) \delta_{y}\left(S_{s}^{-1} \delta_{\bar{z}}\left(K_{\bar{z}}\left(C_{h}^{n}\right) \delta_{z} d_{t} H_{h}^{n}\right)\right)\right)\right)\right)\right)_{i j k}, 1 \leq i, j, k \leq N-1, \\
& H_{h, i j k}^{n+1}=h_{i j k}^{n+1}, X_{i j k} \in \partial \Omega_{h} .
\end{aligned}
$$

From Equation (1) $\left(t=t^{n+1}\right)$ and (18) we have the fluid error equations.

$$
\begin{aligned}
& S_{s, i j k} \frac{\pi_{i j k}^{n+1}-\pi_{i j k}^{n}}{\Delta t}-\left\{\delta_{\bar{x}}\left(K_{1}\left(C_{h}^{n}\right) \delta_{x} \pi^{n+1}\right)_{i j k}+\delta_{\bar{y}}\left(K_{2}\left(C_{h}^{n}\right) \delta_{y} \pi^{n+1}\right)_{i j k}+\delta_{\bar{z}}\left(K_{3}\left(C_{h}^{n}\right) \delta_{z} \pi^{n+1}\right)_{i j k}\right\} \\
& =\nabla_{h}\left(\left[K\left(c^{n+1}\right)-K\left(C_{h}^{n}\right)\right] \nabla_{h} H^{n+1}\right)_{i j k}-\eta \psi_{i j k} \frac{\xi_{i j k}^{n+1}-\xi_{i j k}^{n}}{\Delta t}+\left[\frac{\rho\left(c_{i j k}^{n+1}\right)}{\rho_{0}} q_{i j k}^{n+1}-\frac{\rho\left(C_{h, j k}^{n}\right)}{\rho_{0}} q_{i j k}^{n}\right] \\
& +\eta\left[\delta_{\bar{z}}\left(K_{3}\left(c^{n+1}\right) c^{n+1}\right)_{i j k}-\delta_{\bar{z}}\left(K_{3}\left(C_{h}^{n}\right) C_{h}^{n}\right)_{i j k}\right] \\
& -(\Delta t)^{2}\left\{\left[\delta_{\bar{x}}\left(K_{1}\left(c^{n+1}\right) \delta_{x}\left(S_{s}^{-1} \delta_{\bar{y}}\left(K_{2}\left(c^{n+1}\right) \delta_{y} d_{t} H^{n}\right)\right)\right)_{i j k}\right.\right. \\
& \left.-\delta_{\bar{x}}\left(K_{1}\left(C_{h}^{n}\right) \delta_{x}\left(S_{s}^{-1} \delta_{\bar{y}}\left(K_{2}\left(C_{h}^{n}\right) \delta_{y} d_{t} H_{h}^{n}\right)\right)\right)_{i j k}\right]+\left[\delta_{\bar{x}}\left(K_{1}\left(c^{n+1}\right) \delta_{x}\left(S_{s}^{-1} \delta_{\bar{z}}\left(K_{3}\left(c^{n+1}\right) \delta_{z} d_{t} H^{n}\right)\right)\right)_{i j k}\right. \\
& \left.-\delta_{\bar{x}}\left(K_{1}\left(C_{h}^{n}\right) \delta_{x}\left(S_{s}^{-1} \delta_{\bar{z}}\left(K_{3}\left(C_{h}^{n}\right) \delta_{z} d_{t} H_{h}^{n}\right)\right)\right)_{i j k}\right]+\left[\delta_{\bar{y}}\left(K_{2}\left(c^{n+1}\right) \delta_{y}\left(S_{s}^{-1} \delta_{\bar{z}}\left(K_{\bar{z}}\left(c^{n+1}\right) \delta_{z} d_{t} H^{n}\right)\right)\right)_{i j k}\right. \\
& \left.\left.-\delta_{\bar{y}}\left(K_{2}\left(C_{h}^{n}\right) \delta_{y}\left(S_{s}^{-1} \delta_{\bar{z}}\left(K_{3}\left(C_{h}^{n}\right) \delta_{z} d_{t} H_{h}^{n}\right)\right)\right)_{i j k}\right]\right\} \\
& +(\Delta t)^{3}\left\{\delta_{\bar{x}}\left(K_{1}\left(c^{n+1}\right) \delta_{x}\left(S_{s}^{-1} \delta_{\bar{y}}\left(K_{2}\left(c^{n+1}\right) \delta_{y}\left(S_{s}^{-1} \delta_{\bar{z}}\left(K_{3}\left(c^{n+1}\right) \delta_{z} d_{t} H^{n}\right)\right)\right)\right)\right)_{i j k}\right. \\
& \left.-\delta_{\bar{x}}\left(K_{1}\left(C_{h}^{n}\right) \delta_{x}\left(S_{s}^{-1} \delta_{\bar{y}}\left(K_{2}\left(C_{h}^{n}\right) \delta_{y}\left(S_{s}^{-1} \delta_{\bar{z}}\left(K_{3}\left(C_{h}^{n}\right) \delta_{z} d_{t} H_{h}^{n}\right)\right)\right)\right)\right)_{i j k}\right\}+\varepsilon_{2, j i k}^{n+1},
\end{aligned}
$$

$1 \leq i, j, k \leq N-1$,

$$
\pi_{i j k}^{n+1}=0, X_{i j k} \in \partial \Omega_{h},
$$

where $\left|\varepsilon_{2, j k}^{n+1}\right| \leq M\left(\Delta t+h^{2}\right)$.

We shall introduce the induction hypothesis:

$$
\sup _{0 \leq n \leq L} \max \left\{\left\|\pi^{n}\right\|_{1, \infty},\left\|\xi^{n}\right\|_{1, \infty}\right\} \rightarrow 0,(h, \Delta t) \rightarrow 0,
$$

where

$$
\left\|\pi^{n}\right\|_{1, \infty}^{2}=\left\|\pi^{n}\right\|_{0, \infty}^{2}+\left\|\nabla_{h} \pi^{n}\right\|_{0, \infty}^{2}, \cdots .
$$

We consider fluid error Equation (19). Test error Equation (19) against $\delta_{t} \pi_{i j k}^{n+1}=\pi_{i j k}^{n+1}-\pi_{i j k}^{n}$ and summing it up by parts, we have 


$$
\begin{aligned}
& \left\langle S_{s} d_{t} \pi^{n}, d_{t} \pi^{n}\right\rangle \Delta t+\frac{1}{2}\left\{\left[\left\langle K_{1}\left(C_{h}^{n}\right) \delta_{x} \pi^{n+1}, \delta_{x} \pi^{n+1}\right\rangle+\left\langle K_{2}\left(C_{h}^{n}\right) \delta_{y} \pi^{n+1}, \delta_{y} \pi^{n+1}\right\rangle\right.\right. \\
& \left.\left.+\left\langle K_{3}\left(C_{h}^{n}\right) \delta_{z} \pi^{n+1}, \delta_{z} \pi^{n+1}\right\rangle\right]-\left[\left\langle K_{1}\left(C_{h}^{n}\right) \delta_{x} \pi^{n}, \delta_{x} \pi^{n}\right\rangle\left\langle K_{2}\left(C_{h}^{n}\right) \delta_{y} \pi^{n}, \delta_{y} \pi^{n}\right\rangle+\left\langle K_{3}\left(C_{h}^{n}\right) \delta_{z} \pi^{n}, \delta_{z} \pi^{n}\right\rangle\right]\right\} \\
& \leq\left\langle\nabla_{h}\left(\left[K\left(c^{n+1}\right)-K\left(C_{h}^{n}\right)\right] \nabla_{h} H^{n+1}\right), d_{t} \pi^{n}\right\rangle \Delta t-\eta\left\langle\psi d_{t} \xi^{n}, d_{t} \pi^{n}\right\rangle \Delta t \\
& +\left\langle\frac{\rho\left(c^{n+1}\right)}{\rho_{0}} q^{n+1}-\frac{\rho\left(C_{h}^{n}\right)}{\rho_{0}} q^{n}, d_{t} \pi^{n}\right\rangle \Delta t \\
& +\eta\left\langle\delta_{\bar{z}}\left(K_{3}\left(c^{n+1}\right) c^{n+1}\right)-\delta_{\bar{z}}\left(K_{3}\left(C_{h}^{n}\right) C_{h}^{n}\right), d_{t} \pi^{n}\right\rangle \Delta t \\
& -(\Delta t)^{2}\left\{\left\langle\delta_{\bar{x}}\left(K_{1}\left(c^{n+1}\right)\right) \delta_{x}\left(S_{s}^{-1} \delta_{\bar{y}}\left(K_{2}\left(c^{n+1}\right) \delta_{y} d_{t} H^{n}\right)\right)\right)\right. \\
& \left.-\delta_{\bar{x}}\left(K_{1}\left(C_{h}^{n}\right) \delta_{x}\left(S_{s}^{-1} \delta_{\bar{y}}\left(K_{2}\left(C_{h}^{n}\right) \delta_{y} d_{t} H_{h}^{n}\right)\right)\right), d_{t} \pi^{n}>\Delta t+\cdots\right\} \\
& +(\Delta t)^{3}\left\langle\delta_{\bar{x}}\left(K_{1}\left(c^{n+1}\right) \delta_{x}\left(S_{s}^{-1} \delta_{\bar{y}}\left(K_{2}\left(c^{n+1}\right) \delta_{y}\left(S_{s}^{-1} \delta_{\bar{z}}\left(K_{3}\left(c^{n+1}\right) \delta_{z} d_{t} H^{n}\right)\right)\right)\right)\right)\right. \\
& \left.-\delta_{\bar{x}}\left(K_{1}\left(C_{h}^{n}\right) \delta_{x}\left(S_{s}^{-1} \delta_{\bar{y}}\left(K_{2}\left(C_{h}^{n}\right) \delta_{y}\left(S_{s}^{-1} \delta_{\bar{z}}\left(K_{\bar{z}}\left(C_{h}^{n}\right) \delta_{z} d_{t} H_{h}^{n}\right)\right)\right)\right)\right), d_{t} \pi^{n}\right\rangle \Delta t+\left\langle\varepsilon_{2}^{n+1}, d_{t} \pi^{n}\right\rangle \Delta t .
\end{aligned}
$$

Now we estimate the terms on the right-hand side of (21).

$$
\begin{aligned}
& <\nabla_{h}\left(\left[K\left(c^{n+1}\right)-K\left(C_{h}^{n}\right)\right] \nabla_{h} H^{n+1}\right), d_{t} \pi^{n}>\Delta t \\
& \leq M\left\{\left\|\nabla_{h} \xi^{n}\right\|^{2}+(\Delta t)\right\}^{2} \Delta t+\varepsilon\left\|d_{t} \pi^{n}\right\|^{2} \Delta t, \\
& -\eta\left\langle\psi d_{t} \xi^{n}, d_{t} \pi^{n}\right\rangle \Delta t \\
& \leq M\left\|d_{t} \xi^{n}\right\|^{2} \Delta t+\varepsilon\left\|d_{t} \pi^{n}\right\|^{2} \Delta t,
\end{aligned}
$$

$$
\begin{gathered}
<\frac{\rho\left(c^{n+1}\right)}{\rho_{0}} q^{n+1}-\frac{\rho\left(C_{h}^{n}\right)}{\rho_{0}} q^{n}, d_{t} \pi^{n}>\Delta t \\
\leq M\left\{\left\|\xi^{n}\right\|^{2}+(\Delta t)^{2}\right\} \Delta t+\varepsilon\left\|d_{t} \pi^{n}\right\|^{2} \Delta t, \\
\eta\left\langle\delta_{\bar{z}}\left(K_{3}\left(c^{n+1}\right) c^{n+1}\right)-\delta_{\bar{z}}\left(K_{3}\left(C_{h}^{n}\right) C_{h}^{n}\right), d_{t} \pi^{n}\right\rangle \Delta t \\
\leq M\left\{\left\|\nabla_{h} \xi^{n}\right\|^{2}+\left\|\xi^{n}\right\|^{2}+(\Delta t)^{2}\right\} \Delta t+\varepsilon\left\|d_{t} \pi^{n}\right\|^{2} \Delta t,
\end{gathered}
$$

For the fifth term on the right-hand side of (21).

$$
\begin{aligned}
& -(\Delta t)^{3}<\delta_{\bar{x}}\left(K_{1}\left(c^{n+1}\right) \delta_{x}\left(S_{s}^{-1} \delta_{\bar{y}}\left(K_{2}\left(c^{n+1}\right) \delta_{y}\left(S_{s}^{-1} \delta_{\bar{z}}\left(K_{3}\left(c^{n+1}\right) \delta_{z} d_{t} H^{n}\right)\right)\right)\right)\right) \\
& -\delta_{\bar{x}}\left(K_{1}\left(C_{h}^{n}\right) \delta_{x}\left(S_{s}^{-1} \delta_{\bar{y}}\left(K_{2}\left(C_{h}^{n}\right) \delta_{y}\left(S_{s}^{-1} \delta_{\bar{z}}\left(K_{3}\left(C_{h}^{n}\right) \delta_{z} d_{t} H_{h}^{n}\right)\right)\right)\right)\right), d_{t} \pi^{n}>\Delta t \\
& =-(\Delta t)^{3}\left\{\left\langle\delta_{\bar{x}}\left(K_{1}\left(C_{h}^{n}\right) \delta_{x}\left(S_{s}^{-1} \delta_{\bar{y}}\left(K_{2}\left(C_{h}^{n}\right) \delta_{y} d_{t} \pi^{n}\right)\right)\right), d_{t} \pi^{n}\right\rangle\right. \\
& +\left\langle\delta_{\bar{x}}\left(K_{1}\left(C_{h}^{n}\right) \delta_{x}\left(S_{s}^{-1} \delta_{\bar{y}}\left(\left[K_{2}\left(c^{n+1}\right)-K_{2}\left(C_{h}^{n}\right)\right] \delta_{y} d_{t} H^{n}\right)\right)\right), d_{t} \pi^{n}\right\rangle \\
& \left.+\left\langle\delta_{\bar{x}}\left(\left[K_{1}\left(c^{n+1}\right)-K_{1}\left(C_{h}^{n}\right)\right] \delta_{x}\left(S_{s}^{-1} \delta_{\bar{y}}\left(K_{2}\left(c^{n+1}\right) \delta_{y} d_{t} H^{n}\right)\right)\right), d_{t} \pi^{n}\right\rangle\right\} .
\end{aligned}
$$

For the first term on the right-hand side of (23), though the operators $-\delta_{\bar{x}}\left(K \delta_{x}\right),-\delta_{\bar{y}}\left(K \delta_{y}\right) \cdots$ are self-conjugate, positive definite and bounded, space region is cubic. However, their products are generally incommutative.

\section{Noting that}

$$
\delta_{x} \delta_{y}=\delta_{y} \delta_{x}, \delta_{x} \delta_{\bar{y}}=\delta_{\bar{y}} \delta_{x}, \delta_{\bar{x}} \delta_{y}=\delta_{y} \delta_{\bar{x}}, \delta_{\bar{x}} \delta_{\bar{y}}=\delta_{\bar{y}} \delta_{\bar{x}},
$$

we have 


$$
\begin{aligned}
& -(\Delta t)^{3}\left\{\left\langle\delta_{\bar{x}}\left(K_{1}\left(C_{h}^{n}\right) \delta_{x}\left(S_{s}^{-1} \delta_{\bar{y}}\left(K_{2}\left(C_{h}^{n}\right) \delta_{y} d_{t} \pi^{n}\right)\right)\right), d_{t} \pi^{n}\right\rangle\right. \\
& =-(\Delta t)^{3} \sum_{i, j, k=1}^{N}\left\{K_{1}\left(C_{h}^{n}\right)_{i+1 / 2, j k} K_{2}\left(C_{h}^{n}\right)_{i, j+1 / 2, k} S_{s, i j k}^{-1}\left[\delta_{x} \delta_{y} d_{t} \pi_{i j k}^{n}\right]^{2}+\left[K_{2}\left(C_{h}^{n}\right)_{i, j+1 / 2, k} \cdot \delta_{y}\left(S_{s, i j k}^{-1} K_{1}\left(C_{h}^{n}\right)_{i+1 / 2, j k}\right) \delta_{x} d_{t} \pi_{i j k}^{n}\right.\right. \\
& \left.+S_{s, j k}^{-1} K_{1}\left(C_{h}^{n}\right)_{i+1 / 2, j k} \cdot \delta_{x} K_{2}\left(C_{h}^{n}\right)_{i, j+1 / 2, k} \cdot \delta_{y} d_{t} \pi_{i j k}^{n}+K_{2}\left(C_{h}^{n}\right)_{i, j+1 / 2, k} K_{1}\left(C_{h}^{n}\right)_{i+1 / 2, j k} \cdot \delta_{x} S_{s, i j k}^{-1}\right] \delta_{x} \delta_{y} d_{t} \pi_{i j k}^{n} \\
& +\left[\delta_{x} K_{2}\left(C_{h}^{n}\right)_{i, j+1 / 2, k} \cdot \delta_{y}\left(S_{s, i j k}^{-1} K_{1}\left(C_{h}^{n}\right)_{i+1 / 2, j k}\right)+K_{2}\left(C_{h}^{n}\right)_{i, j+1 / 2, k} \cdot\right. \\
& \left.\left.\delta_{y}\left(\delta_{x} S_{s, j i k}^{-1} \cdot K_{1}\left(C_{h}^{n}\right)_{i+1 / 2, j k}\right)\right] \delta_{x} d_{t} \pi_{i j k}^{n} \cdot \delta_{y} d_{t} \pi_{i j k}^{n}\right\} h^{3} .
\end{aligned}
$$

From induction hypothesis (20) we learn that

$K_{1}\left(C_{h}^{n}\right), \quad K_{2}\left(C_{h}^{n}\right), \quad \delta_{x} K_{1}\left(C_{h}^{n}\right), \delta_{y}\left(S_{s}^{-1} K_{1}\left(C_{h}^{n}\right)\right) \cdots \quad$ are bounded. To the first and second terms of expression
(24), the positive definite property of $K_{1}, K_{2}, S_{s}^{-1}$ should be applied, and high-order difference term $\delta_{x} \delta_{y} d_{t} \pi_{i j k}^{n}$ should be separated. By using Cauchy inequality to eliminate the terms concerned, we can get

$$
\begin{aligned}
& -(\Delta t)^{3} \sum_{i, j, k=1}^{N}\left\{\left\{K_{1}\left(C_{h}^{n}\right)_{i+1 / 2, j k} K_{2}\left(C_{h}^{n}\right)_{i, j+1 / 2, k} S_{s, i j k}^{-1}\left[\delta_{x} \delta_{y} d_{t} \pi_{i j k}^{n}\right]^{2}+\left[K_{2}\left(C_{h}^{n}\right)_{i, j+1 / 2, k}\right.\right.\right. \\
& \cdot \delta_{y}\left(S_{s, i j k}^{-1} K_{1}\left(C_{h}^{n}\right)_{i+1 / 2, j k}\right) \delta_{x} d_{t} \pi_{i j k}^{n}+S_{s, j i k}^{-1} K_{1}\left(C_{h}^{n}\right)_{i+1 / 2, j k} \cdot \delta_{x} K_{2}\left(C_{h}^{n}\right)_{i, j+1 / 2, k} \cdot \delta_{y} d_{t} \pi_{i j k}^{n} \\
& \left.\left.+K_{2}\left(C_{h}^{n}\right)_{i, j+1 / 2, k} K_{1}\left(C_{h}^{n}\right)_{i+1 / 2, j k} \cdot \delta_{x} S_{s, i j k}^{-1}\right] \delta_{x} \delta_{y} d_{t} \pi_{i j k}^{n}\right\} h^{3} \leq-\frac{1}{2} K_{*}^{2}\left(S_{s}^{*}\right)^{-1}(\Delta t)^{3} \sum_{i, j, k=1}^{N}\left[\delta_{x} \delta_{y} d_{t} \pi_{i j k}^{n}\right]^{2} h^{3} \\
& +M\left\{\left\|\delta_{x} d_{t} \pi^{n}\right\|^{2}+\left\|\delta_{y} d_{t} \pi^{n}\right\|^{2}\right\}(\Delta t)^{3} \leq-\frac{1}{2} K_{*}^{2}\left(S_{s}^{*}\right)^{-1}(\Delta t)^{3} \sum_{i, j, k=1}^{N}\left[\delta_{x} \delta_{y} d_{t} \pi_{i j k}^{n}\right]^{2} h^{3}+M\left\{\left\|\nabla_{h} \pi^{n+1}\right\|^{2}+\left\|\nabla_{h} \pi^{n}\right\|^{2}\right\} \Delta t .
\end{aligned}
$$

For the third term of (24), we have

$$
\begin{aligned}
& -(\Delta t)^{3} \sum_{i, j, k=1}^{N}\left\{\left[\delta_{x} K_{2}\left(C_{h}^{n}\right)_{i, j+1 / 2, k} \cdot \delta_{y}\left(S_{s, j i k}^{-1} K_{1}\left(C_{h}^{n}\right)_{i+1 / 2, j k}\right)+K_{2}\left(C_{h}^{n}\right)_{i, j+1 / 2, k}\right.\right. \\
& \left.\left.\cdot \delta_{y}\left(\delta_{x} S_{s, i j k}^{-1} \cdot K_{1}\left(C_{h}^{n}\right)_{i+1 / 2, j k}\right)\right] \delta_{x} d_{t} \pi_{i j k}^{n} \cdot \delta_{y} d_{t} \pi_{i j k}^{n}\right\} h^{3} \\
& \leq M\left\{\left\|\nabla_{h} \pi^{n+1}\right\|^{2}+\left\|\nabla_{h} \pi^{n}\right\|^{2}\right\} \Delta t .
\end{aligned}
$$

Similarly, for the other terms, we can obtain

$$
\begin{aligned}
& -(\Delta t)^{3}\left\{\left\langle\delta_{\bar{x}}\left(K_{1}\left(c^{n+1}\right) \delta_{x}\left(S_{s}^{-1} \delta_{\bar{y}}\left(K_{2}\left(c^{n+1}\right) \delta_{y} d_{t} H^{n}\right)\right)\right)-\delta_{\bar{x}}\left(K_{1}\left(C_{h}^{n}\right) \delta_{x}\left(S_{s}^{-1} \delta_{\bar{y}}\left(K_{2}\left(C_{h}^{n}\right) \delta_{y} d_{t} H_{h}^{n}\right)\right)\right), d_{t} \pi^{n}\right\rangle+\cdots\right. \\
& \left.+\left\langle\delta_{\bar{y}}\left(K_{2}\left(c^{n+1}\right) \delta_{y}\left(S_{s}^{-1} \delta_{\bar{z}}\left(K_{2}\left(c^{n+1}\right) \delta_{z} d_{t} H^{n}\right)\right)\right), \cdots\right\rangle\right\} \leq M\left\{\left\|\nabla_{h} \pi^{n+1}\right\|^{2}+\left\|\nabla_{h} \pi^{n}\right\|^{2}+\left\|\xi^{n}\right\|^{2}+(\Delta t)^{2}\right\} \Delta t .
\end{aligned}
$$

Now, we consider the sixth term of the right-hand side of (21).

$$
\begin{aligned}
& (\Delta t)^{4}\left\langle\delta_{\bar{x}}\left(K_{1}\left(c^{n+1}\right) \delta_{x}\left(S_{s}^{-1} \delta_{\bar{y}}\left(K_{2}\left(c^{n+1}\right) \delta_{y}\left(S_{s}^{-1} \delta_{\bar{z}}\left(K_{3}\left(c^{n+1}\right) \delta_{z} d_{t} H^{n}\right)\right)\right)\right)\right)\right. \\
& \left.-\delta_{\bar{x}}\left(K_{1}\left(C_{h}^{n}\right) \delta_{x}\left(S_{s}^{-1} \delta_{\bar{y}}\left(K_{2}\left(C_{h}^{n}\right) \delta_{y}\left(S_{s}^{-1} \delta_{\bar{z}}\left(K_{3}\left(C_{h}^{n}\right) \delta_{z} d_{t} H_{h}^{n}\right)\right)\right)\right)\right), d_{t} \pi^{n}\right\rangle \\
& \leq-\frac{1}{2} K_{*}^{3}\left(S_{s}^{*}\right)^{-2}(\Delta t)^{4} \sum_{i, j, k=1}^{N}\left[\delta_{x} \delta_{y} \delta_{z} d_{t} \pi_{i j k}^{n}\right]^{2} h^{3}+M\left\{\left\|\nabla_{h} \pi^{n+1}\right\|^{2}+\left\|\nabla_{h} \pi^{n}\right\|^{2}+\left\|\xi^{n}\right\|^{2}+(\Delta t)^{2}\right\} \Delta t .
\end{aligned}
$$


For the last term on the right-hand side of (21).

$$
\left\langle\varepsilon_{2}^{n+1}, d_{t} \pi^{n}\right\rangle \Delta t \leq M\left\{\left\lfloor\nabla_{h} \pi^{n+1}\right\rfloor^{2}+\left\|\nabla_{h} \pi^{n}\right\|^{2}+\left\|\xi^{n}\right\|^{2}+(\Delta t)^{2}\right\} \Delta t .
$$

From (21)-(28) we can obtain

$$
\begin{aligned}
& S_{s}^{*}\left\|d_{t} \pi^{n}\right\|^{2} \Delta t+\frac{1}{2}\left\{\left\langle K\left(C_{h}^{n}\right) \nabla_{h} \pi^{n+1}, \nabla_{h} \pi^{n+1}\right\rangle-\left\langle K\left(C_{h}^{n}\right) \nabla_{h} \pi^{n}, \nabla_{h} \pi^{n}\right\rangle\right\} \\
& \leq M\left\{\left\|\nabla_{h} \pi^{n+1}\right\|^{2}+\left\|\nabla_{h} \pi^{n}\right\|^{2}+\left\|\xi^{n}\right\|^{2}+\left\|\nabla_{h} \xi^{n}\right\|^{2}+\left\|d_{h} \xi^{n}\right\|^{2}+h^{4}+(\Delta t)^{2}\right\} \Delta t+\varepsilon\left\|d_{t} \pi^{n}\right\|^{2} \Delta t .
\end{aligned}
$$

Next, consider the concentration error equation. Test ing it up by parts, we have error Equation (17) against $\delta_{t} \xi_{i j k}^{n+1}=\xi_{i j k}^{n+1}-\xi_{i j k}^{n}$ and sum-

$$
\begin{aligned}
& \left\langle\beta\left(C_{h}^{n}\right) d_{t} \xi^{n}, d_{t} \xi^{n}\right\rangle \Delta t+\left\{\left\langle\bar{D}_{1} \delta_{x} \xi^{n+1}, \delta_{x}\left[\left(1+\frac{h}{2}\left|u_{1}^{n+1}\right| \bar{D}_{1}^{-1}\right)^{-1}\left(\xi^{n+1}-\xi^{n}\right)\right]\right\rangle\right. \\
& +\left\langle\bar{D}_{2} \delta_{y} \xi^{n+1}, \delta_{y}\left[\left(1+\frac{h}{2}\left|u_{2}^{n+1}\right| \bar{D}_{2}^{-1}\right)^{-1}\left(\xi^{n+1}-\xi^{n}\right)\right]\right\rangle+\left\langle\bar{D}_{3} \delta_{z} \xi^{n+1}, \delta_{z}\left[\left(1+\frac{h}{2}\left|u_{3}^{n+1}\right| \bar{D}_{1}^{-1}\right)^{-1}\left(\xi^{n+1}-\xi^{n}\right)\right]\right\rangle \\
& \left.=\left\langle\delta_{U_{1}^{n}, x} C_{h}^{n}-\delta_{U_{1}^{n}, x} c^{n+1}, d_{t} \xi^{n}\right\rangle+\left\langle\delta_{U_{2}^{n}, y} C_{h}^{n}-\delta_{U_{2}^{n}, y} c^{n+1}, d_{t} \xi^{n}\right\rangle+\left\langle\delta_{U_{3}^{n}, z} C_{h}^{n}-\delta_{U_{3}^{n}, z} c^{n+1}, d_{t} \xi^{n}\right\rangle\right\} \Delta t \\
& +\left\{\left\langle\left[\left(1+\frac{h}{2}\left|u_{1}^{n+1}\right| \bar{D}_{1}^{-1}\right)^{-1}-\left(1+\frac{h}{2}\left|u_{1}^{n}\right| \bar{D}_{1}^{-1}\right)^{-1}\right] \delta_{\bar{x}}\left(\bar{D}_{1} \delta_{x} C_{h}^{n+1}\right), d_{t} \xi^{n}\right\rangle\right. \\
& \left\langle\left[\left(1+\frac{h}{2}\left|u_{2}^{n+1}\right| \bar{D}_{1}^{-1}\right)^{-1}-\left(1+\frac{h}{2}\left|u_{2}^{n}\right| \bar{D}_{1}^{-1}\right)^{-1}\right] \delta_{\bar{y}}\left(\bar{D}_{1} \delta_{y} C_{h}^{n+1}\right), d_{t} \xi^{n}\right\rangle \\
& \left.\left\langle\left[\left(1+\frac{h}{2}\left|u_{3}^{n+1}\right| \bar{D}_{1}^{-1}\right)^{-1}-\left(1+\frac{h}{2}\left|u_{3}^{n}\right| \bar{D}_{1}^{-1}\right)^{-1}\right] \delta_{\bar{z}}\left(\bar{D}_{1} \delta_{z} C_{h}^{n+1}\right), d_{t} \xi^{n}\right\rangle\right\} \Delta t \\
& +\left\langle q^{n+1}\left(C^{*, n+1}-C^{n+1}\right)-q^{n}\left(C^{*, n}-C^{n}\right), d_{t} \xi^{n}\right\rangle \Delta t \\
& +(\Delta t)^{3}\left\{\left\langle\left(1+\frac{h}{2}\left|u_{1}^{n+1}\right| \bar{D}_{1}^{-1}\right)^{-1} \delta_{\bar{x}}\left(\bar{D}_{1} \delta_{x}\left(\beta^{-1}\left(C_{h}^{n}\right)\left(1+\frac{h}{2}\left|u_{3}^{n}\right| \bar{D}_{3}^{-1}\right)^{-1} \delta_{\bar{z}}\left(\bar{D}_{1} \delta_{z} d_{t} \xi^{n}\right)\right)\right), d_{t} \xi^{n}\right\rangle+\cdots\right\} \\
& +(\Delta t)^{4}\left(1+\frac{h}{2}\left|u_{1}^{n}\right| \bar{D}_{1}^{-1}\right)^{-1} \\
& \delta_{\bar{x}}\left(\bar{D}_{1} \delta_{x}\left(\beta^{-1}\left(C_{h}^{n}\right)\left(1+\frac{h}{2}\left|u_{3}^{n}\right| \bar{D}_{3}^{-1}\right)^{-1} \delta_{\bar{y}}\left(\bar{D}_{2} \delta_{y}\left(\beta^{-1}\left(C_{h}^{n}\right)\left(1+\frac{h}{2}\left|u_{3}^{n}\right| \bar{D}_{3}^{-1} \xi^{n}\right\rangle \Delta t \delta_{\bar{z}}\left(\bar{D}_{3} \delta_{z} d_{t} \xi^{n}\right)\right)\right)\right),+\cdots\right\}
\end{aligned}
$$

First, we estimate the second term on the left-hand side of (30).

$$
\begin{aligned}
& \left\langle\bar{D}_{1} \delta_{x} \xi^{n+1}, \delta_{x}\left[\left(1+\frac{h}{2}\left|u_{1}^{n+1}\right| \bar{D}_{1}^{-1}\right)^{-1}\left(\xi^{n+1}-\xi^{n}\right)\right]\right\rangle \geq \frac{1}{2}\left\{\left\langle\bar{D}_{1} \delta_{x} \xi^{n+1},\left(1+\frac{h}{2}\left|u_{1}^{n+1}\right| \bar{D}_{1}^{-1}\right)^{-1} \delta_{x} \xi^{n+1}\right\rangle\right. \\
& \left.-\left\langle\bar{D}_{1} \delta_{x} \xi^{n},\left(1+\frac{h}{2}\left|u_{1}^{n+1}\right| \bar{D}_{1}^{-1}\right)^{-1} \delta_{x} \xi^{n}\right\rangle\right\}-M\left\|\nabla_{h} \xi^{n+1}\right\|^{2} \Delta t-\varepsilon\left\|d_{t} \xi^{n}\right\|^{2} \Delta t .
\end{aligned}
$$


Similarly,

$$
\begin{aligned}
& \left\langle\bar{D}_{2} \delta_{y} \xi^{n+1}, \delta_{y}\left[\left(1+\frac{h}{2}\left|u_{2}^{n+1}\right| \bar{D}_{2}^{-1}\right)^{-1}\left(\xi^{n+1}-\xi^{n}\right)\right]\right\rangle \\
& \geq \frac{1}{2}\left\{\left\langle\bar{D}_{2} \delta_{y} \xi^{n+1},\left(1+\frac{h}{2}\left|u_{2}^{n+1}\right| \bar{D}_{2}^{-1}\right)^{-1} \delta_{y} \xi^{n+1}\right\rangle-\left\langle\bar{D}_{2} \delta_{y} \xi^{n},\left(1+\frac{h}{2}\left|u_{2}^{n+1}\right| \bar{D}_{2}^{-1}\right)^{-1} \delta_{y} \xi^{n}\right\rangle\right\}-M\left\|\nabla_{h} \xi^{n+1}\right\|^{2} \Delta t-\varepsilon\left\|d_{t} \xi^{n}\right\|^{2} \Delta t . \\
& \left\langle\bar{D}_{3} \delta_{z} \xi^{n+1}, \delta_{z}\left[\left(1+\frac{h}{2}\left|u_{3}^{n+1}\right| \bar{D}_{3}^{-1}\right)^{-1}\left(\xi^{n+1}-\xi^{n}\right)\right]\right\rangle \\
& \geq \frac{1}{2}\left\{\left\langle\bar{D}_{3} \delta_{z} \xi^{n+1},\left(1+\frac{h}{2}\left|u_{3}^{n+1}\right| \bar{D}_{3}^{-1}\right)^{-1} \delta_{z} \xi^{n+1}\right\rangle-\left\langle\bar{D}_{3} \delta_{z} \xi^{n},\left(1+\frac{h}{2}\left|u_{3}^{n+1}\right| \bar{D}_{3}^{-1}\right)^{-1} \delta_{z} \xi^{n}\right\rangle\right\}-M\left\|\nabla_{h} \xi^{n+1}\right\|^{2} \Delta t-\varepsilon\left\|d_{t} \xi^{n}\right\|^{2} \Delta t .
\end{aligned}
$$

Now, we estimate the terms on the right-hand side of (30). In induction hypothesis (20) $U^{n}$ is bounded, so we have

$$
\left\langle\delta_{U_{1}^{n}, x} C_{h}^{n}-\delta_{u_{1}^{n+1}, x} c^{n+1}, d_{t} \xi^{n}\right\rangle \Delta t \leq M\left\{\left\|U_{1}^{n}-u_{1}^{n}\right\|^{2}+\left\|\nabla_{h} \xi^{n}\right\|^{2}+(\Delta t)^{2}\right\} \Delta t+\varepsilon\left\|d_{t} \xi^{n}\right\|^{2} \Delta t .
$$

Similarly,

$$
\begin{aligned}
& \left\{\left\langle\delta_{U^{n}, y} C_{h}^{n}-\delta_{u_{2}^{n+1}, y} c^{n+1}, d_{t} \xi^{n}\right\rangle+\left\langle\delta_{U_{3}^{n}, z} C_{h}^{n}-\delta_{u_{3}^{n+1}, z} c^{n+1}, d_{t} \xi^{n}\right\rangle\right\} \Delta t \\
& \leq M\left\{\left\|U_{2}^{n}-u_{2}^{n}\right\|^{2}+\left\|U_{3}^{n}-u_{3}^{n}\right\|^{2}+\left\|\nabla_{h} \xi^{n}\right\|^{2}+(\Delta t)^{2}\right\} \Delta t+\varepsilon\left\|d_{t} \xi^{n}\right\|^{2} \Delta t .
\end{aligned}
$$

For the second term on the right-hand side of (30), we have

$$
\begin{aligned}
& \left\{\left\langle\left[\left(1+\frac{h}{2}\left|u_{1}^{n+1}\right| \bar{D}_{1}^{-1}\right)^{-1}-\left(1+\frac{h}{2}\left|U_{1}^{n}\right| \bar{D}_{1}^{-1}\right)^{-1}\right] \delta_{\bar{x}}\left(\bar{D}_{1} \delta_{x} C_{h}^{n+1}\right), d_{t} \xi^{n}\right\rangle+\cdots\right. \\
& \left.\left\langle\left[\left(1+\frac{h}{2}\left|u_{3}^{n+1}\right| \bar{D}_{3}^{-1}\right)^{-1}-\left(1+\frac{h}{2}\left|U_{3}^{n}\right| \bar{D}_{3}^{-1}\right)^{-1}\right] \delta_{\bar{z}}\left(\bar{D}_{3} \delta_{z} C_{h}^{n+1}\right), d_{t} \xi^{n}\right\rangle\right\} \Delta t \leq M\left\{\left\|u^{n}-U^{n}\right\|^{2}+(\Delta t)^{2}\right\} \Delta t+\varepsilon\left\|d_{t} \xi^{n}\right\|^{2} \Delta t .
\end{aligned}
$$

For the third term, we have

$$
\left\langle q^{n+1}\left(C^{*, n+1}-c^{n+1}\right)-q^{n}\left(C^{*, n}-C^{n}\right), d_{t} \xi^{n}\right\rangle \Delta t \leq M\left\{\left\|\xi^{n}\right\|^{2}+(\Delta t)^{2}\right\} \Delta t+\varepsilon\left\|d_{t} \xi^{n}\right\|^{2} \Delta t .
$$

We consider the fourth term, and we have

$$
\begin{aligned}
& -(\Delta t)^{3}\left\{\left\langle\left(\left(1+\frac{h}{2}\left|U_{1}^{n}\right| \bar{D}_{1}^{-1}\right)^{-1} \delta_{\bar{x}}\left(\bar{D}_{1} \delta_{x}\left(\beta^{-1}\left(C_{h}^{n}\right)\left(1+\frac{h}{2}\left|U_{2}^{n}\right| \bar{D}_{2}^{-1}\right)^{-1} \delta_{\bar{y}}\left(\bar{D}_{2} \delta_{y} d_{t} \xi^{n}\right)\right), d_{t} \xi^{n}\right\rangle\right.\right.\right. \\
& =-(\Delta t)^{3} \sum_{i, j, k=1}^{N}\left\{\bar{D}_{1, i+1 / 2, j k} \bar{D}_{2, i, j+1 / 2, k} \beta^{-1}\left(C_{h, i j k}^{n}\right)\left(1+\frac{h}{2}\left|U_{1, j i k}^{n}\right| \bar{D}_{1, i j k}^{-1}\right)^{-1}\left(1+\frac{h}{2}\left|U_{2, i j k}^{n}\right| \bar{D}_{2, i j k}^{-1}\right)^{-1}\left(\delta_{x} \delta_{y} d_{t} \xi_{i j k}^{n}\right)^{2}\right. \\
& +\left[\bar{D}_{2, i, j+1 / 2, k} \delta_{x} \bar{D}_{2, i, j+1 / 2, k} \beta^{-1}\left(C_{h, j k}^{n}\right)\left(1+\frac{h}{2}\left|U_{2, i j k}^{n}\right| \bar{D}_{2, i j k}^{-1}\right)^{-1}\left(1+\frac{h}{2}\left|U_{1, j i k}^{n}\right| \bar{D}_{1, i j k}^{-1}\right)^{-1} \delta_{y} d_{t} \xi_{i j k}^{n}+\bar{D}_{2, i, j+1 / 2, k}\right. \\
& \left.\left.\delta_{y}\left[\bar{D}_{1, i+1 / 2, j k} \beta^{-1}\left(C_{h, j k k}^{n}\right)\left(1+\frac{h}{2}\left|U_{2, i j k}^{n}\right| \bar{D}_{2, i j k}^{-1}\right)^{-1}\left(1+\frac{h}{2}\left|U_{1, i j k}^{n}\right| \bar{D}_{1, i j k}^{-1}\right)^{-1}\right] \delta_{x} d_{t} \xi_{i j k}^{n}+\cdots\right] \delta_{x} \delta_{y} d_{t} \xi_{i j k}^{n}+\cdots\right\} h^{3} .
\end{aligned}
$$


On positive definite condition:

$$
\psi_{*} D_{*} \leq \bar{D}_{i} \leq \psi^{*} D^{*}, 0<\beta_{*} \leq \beta(C) \leq \beta^{*}, 0<\left(\beta^{*}\right)^{-1} \leq \beta^{-1}(C) \leq\left(\beta_{*}\right)^{-1} .
$$

In induction hypothesis (20) $U^{n}$ is bounded, so we have $\left(1+h / 2\left|U_{\alpha}^{n}\right| \bar{D}_{\alpha}^{-1}\right)^{-1} \geq b_{0}>0, \alpha=1,2,3$,

$$
\begin{aligned}
& -(\Delta t)^{3}\left\{\left(\left(1+\frac{h}{2}\left|U_{1}^{n}\right| \bar{D}_{1}^{-1}\right)^{-1} \delta_{\bar{x}}\left(\bar{D}_{1} \delta_{x}\left(\beta^{-1}\left(C_{h}^{n}\right)\left(1+\frac{h}{2}\left|U_{2}^{n}\right| \bar{D}_{2}^{-1}\right)^{-1} \delta_{\bar{y}}\left(\bar{D}_{2} \delta_{y} d_{t} \xi^{n}\right)\right), d_{t} \xi^{n}\right\rangle\right.\right. \\
& \leq-\frac{(\Delta t)^{3}}{2}\left(\psi_{*} D_{*}\right)^{2}\left(\beta^{*}\right)^{-1} b_{0}^{2}\left\|\delta_{x} \delta_{y} d_{t} \xi^{n}\right\|^{2}+M(\Delta t)^{3}\left\{\left\|\nabla_{h} d_{t} \xi^{n}\right\|^{2}+\left\|d_{t} \xi^{n}\right\|^{2}\right\} .
\end{aligned}
$$

Thus, for the fourth term we can obtain

$$
\begin{aligned}
& -(\Delta t)^{3}\left\{\left\langle\left(1+\frac{h}{2}\left|U_{1}^{n}\right| \bar{D}_{1}^{-1}\right)^{-1} \delta_{\bar{x}}\left(\bar{D}_{1} \delta_{x}\left(\beta^{-1}\left(C_{h}^{n}\right)\left(1+\frac{h}{2}\left|U_{2}^{n}\right| \bar{D}_{2}^{-1}\right)^{-1} \delta_{\bar{y}}\left(\bar{D}_{2} \delta_{y} d_{t} \xi^{n}\right)\right)\right), d_{t} \xi^{n}\right\rangle+\cdots\right. \\
& \left.+\left\langle\left(\left[1+\frac{h}{2}\left|u_{1}^{n+1}\right| \bar{D}_{1}^{-1}\right)^{-1}-\left(1+\frac{h}{2}\left|U_{1}^{n}\right| \bar{D}_{1}^{-1}\right)^{-1}\right] \delta_{\bar{x}}\left(\bar{D}_{1} \delta_{x}\left(\beta^{-1}\left(c^{n+1}\right)\left(1+\frac{h}{2}\left|u_{2}^{n+1}\right| \bar{D}_{2}^{-1}\right)^{-1} \delta_{\bar{y}}\left(\bar{D}_{2} \delta_{y} d_{t} c^{n}\right)\right)\right), d_{t} \xi^{n}\right\rangle\right\} \\
& \leq-\frac{(\Delta t)^{3}}{2}\left(\psi_{*} D_{*}\right)^{2}\left(\beta^{*}\right)^{-1} b_{0}^{2}\left\|\delta_{x} \delta_{y} d_{t} \xi^{n}\right\|^{2}+M\left\{\left\|\nabla_{h} \xi^{n+1}\right\|^{2}+\left\|\nabla_{h} \xi^{n}\right\|^{2}+\left\|\xi^{n+1}\right\|^{2}+\left\|\xi^{n}\right\|^{2}\right\} \Delta t .
\end{aligned}
$$

Similarly, for the fifth and sixth terms we have

$$
\begin{aligned}
& -(\Delta t)^{3}\left\{\left\langle\left(1+\frac{h}{2}\left|U_{1}^{n}\right| \bar{D}_{1}^{-1}\right)^{-1} \delta_{\bar{x}}\left(\bar{D}_{1} \delta_{x}\left(\beta^{-1}\left(C_{h}^{n}\right)\left(1+\frac{h}{2}\left|U_{3}^{n}\right| \bar{D}_{3}^{-1}\right)^{-1} \delta_{\bar{z}}\left(\bar{D}_{3} \delta_{z} d_{t} \xi^{n}\right)\right), d_{t} \xi^{n}\right\rangle+\cdots\right\}\right. \\
& \leq-\frac{(\Delta t)^{3}}{2}\left(\psi_{*} D_{*}\right)^{2}\left(\beta^{*}\right)^{-1} b_{0}^{2}\left\{\left\|\delta_{x} \delta_{y} d_{t} \xi^{n}\right\|^{2}+\left\|\delta_{x} \delta_{z} d_{t} \xi^{n}\right\|^{2}+\left\|\delta_{y} \delta_{z} d_{t} \xi^{n}\right\|^{2}\right\} \\
& +M\left\{\left\|\nabla_{h} \xi^{n+1}\right\|^{2}+\left\|\nabla_{h} \xi^{n}\right\|^{2}+\left\|\xi^{n+1}\right\|^{2}+\left\|\xi^{n}\right\|^{2}\right\} \Delta t .
\end{aligned}
$$

For the seventh term we have

$$
\begin{aligned}
& (\Delta t)^{4}\left\{\left\langle( 1 + \frac { h } { 2 } | U _ { 1 } ^ { n } | \overline { D } _ { 1 } ^ { - 1 } ) ^ { - 1 } \delta _ { \overline { x } } \left(\overline { D } _ { 1 } \delta _ { x } \left(\beta^{-1}\left(C_{h}^{n}\right)\left(1+\frac{h}{2}\left|U_{2}^{n}\right| \bar{D}_{2}^{-1}\right)^{-1}\right.\right.\right.\right. \\
& \left.\left.\left.\left.\delta_{\bar{y}}\left(\bar{D}_{2} \delta_{y} \beta^{-1}\left(C_{h}^{n}\right)\left(1+\frac{h}{2}\left|U_{3}^{n}\right| \bar{D}_{3}^{-1}\right)^{-1} \delta_{\bar{z}}\left(\bar{D}_{3} \delta_{z} d_{t} \xi^{n}\right)\right)\right)\right)_{i j k}, d_{t} \xi^{n}\right\rangle+\cdots\right\} \\
& \leq-\frac{(\Delta t)^{4}}{2}\left(\psi_{*} D_{*}\right)^{3}\left(\beta^{*}\right)^{-2} b_{0}^{2}\left\|\delta_{x} \delta_{y} \delta_{z} d_{t} \xi^{n}\right\|^{2}+M\left\{\left\|\nabla_{h} \xi^{n+1}\right\|^{2}+\left\|\nabla_{h} \xi^{n}\right\|^{2}+\left\|\xi^{n+1}\right\|^{2}+\left\|\xi^{n}\right\|^{2}\right\} \Delta t .
\end{aligned}
$$

For the last term

$$
\left\langle\varepsilon_{1}^{n+1}, d_{t} \xi^{n}\right\rangle \Delta t \leq \varepsilon\left\|d_{t} \xi^{n}\right\|^{2} \Delta t+M\left\{(\Delta t)^{2}+h^{4}\right\} .
$$

For error Equation (30), from (31)-(37) we can obtain 


$$
\begin{aligned}
& \left\|d_{t} \xi^{n}\right\|^{2} \Delta t+\frac{1}{2}\left\{\left[\left\langle\bar{D}_{1} \delta_{x} \xi^{n+1},\left(1+\frac{h}{2}\left|u_{1}^{n+1}\right| \bar{D}_{1}^{-1}\right)^{-1} \delta_{x} \xi^{n+1}\right\rangle+\left\langle\bar{D}_{2} \delta_{y} \xi^{n+1},\left(1+\frac{h}{2}\left|u_{2}^{n+1}\right| \bar{D}_{2}^{-1}\right)^{-1} \delta_{y} \xi^{n+1}\right\rangle\right.\right. \\
& \left.+\left\langle\bar{D}_{3} \delta_{z} \xi^{n+1},\left(1+\frac{h}{2}\left|u_{3}^{n+1}\right| \bar{D}_{3}^{-1}\right)^{-1} \delta_{z} \xi^{n+1}\right\rangle\right]-\left[\left\langle\bar{D}_{1} \delta_{x} \xi^{n},\left(1+\frac{h}{2}\left|u_{1}^{n+1}\right| \bar{D}_{1}^{-1}\right)^{-1} \delta_{x} \xi^{n}\right\rangle\right. \\
& \left.\left.+\left\langle\bar{D}_{2} \delta_{y} \xi^{n},\left(1+\frac{h}{2}\left|u_{2}^{n+1}\right| \bar{D}_{2}^{-1}\right)^{-1} \delta_{y} \xi^{n}\right\rangle+\left\langle\bar{D}_{3} \delta_{z} \xi^{n},\left(1+\frac{h}{2}\left|u_{3}^{n+1}\right| \bar{D}_{3}^{-1}\right)^{-1} \delta_{z} \xi^{n}\right\rangle\right]\right\} \\
& \leq M\left\{\left\|u^{n}-U^{n}\right\|^{2}+\left\|\nabla_{h} \xi^{n+1}\right\|^{2}+\left\|\nabla_{h} \xi^{n}\right\|^{2}+\left\|\xi^{n+1}\right\|^{2}+\left\|\xi^{n}\right\|^{2}+(\Delta t)^{2}\right\} \Delta t+\varepsilon\left\|d_{t} \xi^{n}\right\|^{2} \Delta t .
\end{aligned}
$$

For fluid error Equation (29), summing over $0 \leq n \leq L$ and noting that $\pi^{0}=0$, we have

$$
\begin{aligned}
& \sum_{n=0}^{L}\left\|d_{t} \pi^{n}\right\|^{2} \Delta t+\left\langle K\left(C_{h}^{L}\right) \nabla_{h} \pi^{L+1}, \nabla_{h} \pi^{L+1}\right\rangle-\left\langle K\left(C_{h}^{0}\right) \nabla_{h} \pi^{0}, \nabla_{h} \pi^{0}\right\rangle \\
& \left.\leq \sum_{n=1}^{L}\left\langle\left[K\left(C_{h}^{n}\right)-K\left(C_{h}^{n-1}\right)\right] \nabla_{h} \pi^{n}, \nabla_{h} \pi^{n}\right\rangle+\sum_{n=0}^{L}\left\|u^{n}-U^{n}\right\|^{2}+\left\|\nabla_{h} \xi^{n+1}\right\|^{2}+\left\|\nabla_{h} \xi^{n}\right\|^{2}+\left\|\xi^{n+1}\right\|^{2}+\left\|\xi^{n}\right\|^{2}+(\Delta t)^{2}\right\} \Delta t
\end{aligned}
$$

For the first term on the right-hand side of (39) we have

$$
\sum_{n=1}^{L}\left\langle\left[K\left(C_{h}^{n}\right)-K\left(C_{h}^{n-1}\right)\right] \nabla_{h} E^{n}, \nabla_{h} \pi^{n}\right\rangle \leq \varepsilon \sum_{n=1}^{L}\left\|d_{t} \xi^{n-1}\right\|^{2} \Delta t+M \sum_{n=1}^{L}\left\|\nabla_{h} \pi^{n}\right\|^{2} \Delta t
$$

By

we have

$$
\left\|u^{n}-U^{n}\right\|^{2} \leq M\left\{\left\|\xi^{n}\right\|^{2}+\left\|\nabla_{h} \pi^{n}\right\|^{2}+h^{4}\right\}
$$

$$
\sum_{n=0}^{L}\left\|d_{t} \pi^{n}\right\|^{2} \Delta t+\left\|\pi^{L+1}\right\|_{1}^{2} \leq \varepsilon \sum_{n=0}^{L-1}\left\|d_{t} \xi^{n}\right\|^{2} \Delta t+M\left\{\sum_{n=1}^{L}\left[\left\|\nabla_{h} \pi^{n}\right\|^{2}+\left\|\xi^{n+1}\right\|_{1}^{2}\right] \Delta t+(\Delta t)^{2}+h^{4}\right\} .
$$

For concentration error Equation (38), summing over $0 \leq n \leq L$ and noting that $\xi^{0}=0$, we can obtain

$$
\begin{aligned}
& \sum_{n=0}^{L}\left\|d_{t} \xi^{n}\right\|^{2} \Delta t+\frac{1}{2}\left\{\left[\left\langle\bar{D}_{1} \delta_{x} \xi^{L+1},\left(1+\frac{h}{2}\left|u_{1}^{L+1}\right| \bar{D}_{1}^{-1}\right)^{-1} \delta_{x} \xi^{L+1}\right\rangle\right.\right. \\
& \left.+\left\langle\bar{D}_{2} \delta_{y} \xi^{L+1},\left(1+\frac{h}{2}\left|u_{2}^{L+1}\right| \bar{D}_{2}^{-1}\right)^{-1} \delta_{y} \xi^{L+1}\right\rangle+\left\langle\bar{D}_{3} \delta_{z} \xi^{L+1},\left(1+\frac{h}{2}\left|u_{3}^{L+1}\right| \bar{D}_{3}^{-1}\right)^{-1} \delta_{z} \xi^{L+1}\right\rangle\right] \\
& -\left[\left\langle\bar{D}_{1} \delta_{x} \xi^{0},\left(1+\frac{h}{2}\left|u_{1}^{0}\right| \bar{D}_{1}^{-1}\right)^{-1} \delta_{x} \xi^{0}\right\rangle+\left\langle\bar{D}_{2} \delta_{y} \xi^{0},\left(1+\frac{h}{2}\left|u_{2}^{0}\right| \bar{D}_{2}^{-1}\right)^{-1} \delta_{y} \xi^{0}\right\rangle+\left\langle\bar{D}_{3} \delta_{z} \xi^{0},\left(1+\frac{h}{2}\left|u_{3}^{0}\right| \bar{D}_{3}^{-1}\right)^{-1} \delta_{z} \xi^{0}\right\rangle\right\} \\
& \leq \frac{1}{2} \sum_{n=1}^{L}\left\{\left\langle\bar{D}_{1} \delta_{x} \xi^{n},\left[\left(1+\frac{h}{2}\left|u_{1}^{n}\right| \bar{D}_{1}^{-1}\right)^{-1}-\left(1+\frac{h}{2}\left|u_{1}^{n-1}\right| \bar{D}_{1}^{-1}\right)^{-1}\right] \delta_{x} \xi^{n}\right\rangle\right. \\
& +\left\langle\bar{D}_{2} \delta_{y} \xi^{n},\left[\left(1+\frac{h}{2}\left|u_{2}^{n}\right| \bar{D}_{2}^{-1}\right)^{-1}-\left(1+\frac{h}{2}\left|u_{2}^{n-1}\right| \bar{D}_{2}^{-1}\right)^{-1}\right] \delta_{y} \xi^{n}\right\rangle \\
& \left.\left\langle\bar{D}_{3} \delta_{z} \xi^{n},\left[\left(1+\frac{h}{2}\left|u_{3}^{n}\right| \bar{D}_{3}^{-1}\right)^{-1}-\left(1+\frac{h}{2}\left|u_{3}^{n-1}\right| \bar{D}_{3}^{-1}\right)^{-1}\right] \delta_{z} \xi^{n}\right\rangle\right\}+M\left\{\sum_{n=0}^{L}\left[\left.\left\|\nabla_{h} \pi^{n}\right\|\right|^{2}+\left\|\xi^{n+1}\right\|_{1}^{2}\right] \Delta t+(\Delta t)^{2}+h^{4}\right\} \Delta t .
\end{aligned}
$$


Then,

$$
\begin{aligned}
& \sum_{n=0}^{L}\left\|d_{t} \xi^{n}\right\|^{2} \Delta t+\frac{1}{2}\left\{\left\langle\bar{D}_{1} \delta_{x} \xi^{L+1},\left(1+\frac{h}{2}\left|u_{1}^{L+1}\right| \bar{D}_{1}^{-1}\right)^{-1} \delta_{x} \xi^{L+1}\right\rangle\right. \\
& \left.+\left\langle\bar{D}_{2} \delta_{y} \xi^{L+1},\left(1+\frac{h}{2}\left|u_{2}^{L+1}\right| \bar{D}_{2}^{-1}\right)^{-1} \delta_{y} \xi^{L+1}\right\rangle+\left\langle\bar{D}_{3} \delta_{z} \xi^{L+1},\left(1+\frac{h}{2}\left|u_{3}^{L+1}\right| \bar{D}_{3}^{-1}\right)^{-1} \delta_{z} \xi^{L+1}\right\rangle\right\} \\
& \leq M\left\{\sum_{n=0}^{L}\left[\left\|\nabla_{h} \pi^{n}\right\|^{2}+\left\|\xi^{n+1}\right\|_{1}^{2}\right] \Delta t+(\Delta t)^{2}+h^{4}\right\} \Delta t .
\end{aligned}
$$

Combining (42) with (43) it yields

$$
\sum_{n=0}^{L}\left[\left\|d_{t} \xi^{n}\right\|^{2}+\left\|d_{t} \pi^{n}\right\|^{2}\right] \Delta t+\left\|\xi^{L+1}\right\|_{1}^{2}+\left\|\pi^{L+1}\right\|_{1}^{2} \leq M\left\{\sum_{n=0}^{L}\left[\left\|\pi^{n}\right\|_{1}^{2}+\left\|\xi^{n+1}\right\|_{1}^{2}\right] \Delta t+(\Delta t)^{2}+h^{4}\right\} \Delta t .
$$

Applying discrete Gronwall inequality, we have

$$
\sum_{n=0}^{L}\left[\left\|d_{t} \xi^{n}\right\|^{2}+\left\|d_{t} \pi^{n}\right\|^{2}\right] \Delta t+\left\|\xi^{L+1}\right\|_{1}^{2}+\left\|\pi^{L+1}\right\|_{1}^{2} \leq M\left\{(\Delta t)^{2}+h^{4}\right\} .
$$

It remains to check induction hypothesis (20). First, for $n=0$, since $\pi^{0}=\xi^{0}=0,(20)$ is correct. If $1 \leq n \leq L$, (20) holds. From (45) we have $\left\|\pi^{L+1}\right\|_{1 \infty}+\left\|\xi^{L+1}\right\|_{1, \infty} \leq M h^{1 / 2}$, then induction hypothesis holds for $n=L+1$.

For the first order weighted upwind finite difference fractional steps scheme, we have the following theorem.

Theorem II. Suppose that exact solutions of problem (1)-(5) satisfy condition:

$$
\begin{gathered}
H, c \in W^{1, \infty}\left(W^{1, \infty}\right) \cap L^{\infty}\left(W^{4, \infty}\right), \\
\partial H / \partial t, \partial c / \partial t \in L^{\infty}\left(W^{4, \infty}\right), \partial^{2} H / \partial t^{2}, \partial^{2} c / \partial t^{2} \in L^{\infty}\left(L^{\infty}\right) .
\end{gathered}
$$

Adopt the first order weighted upwind procedures $(8)^{\prime}-(10)^{\prime},(11)-(13)$. Then the following error estimates hold:

$$
\begin{aligned}
& \left\|H-H_{h}\right\|_{\bar{L}^{\infty}\left(J ; h^{1}\right)}+\left\|c-C_{h}\right\|_{\bar{L}^{\infty}\left(J ; h^{1}\right)}+\left\|d_{t}\left(H-H_{h}\right)\right\|_{\bar{L}^{2}\left(J ; l^{2}\right)} \\
& +\left\|d_{t}\left(c-C_{h}\right)\right\|_{\bar{L}^{2}\left(J ; l^{2}\right)} \leq M^{* *}(\Delta t+h) .
\end{aligned}
$$

\section{Numerical Simulation Results and Analysis}

Considering the complexity of problem, we select Huang- heying area of Longkou city as the model area which has 3-dimensional observation grid. This area is on the left bank of Huangshui River neighboring with Bohai in the north and Huangshui River in the east. Its length is 3000 meters and the width is 700 meters. Its average thickness is about 17 or 18 meters. In the upper part of the water-containing layer there is relatively fine sand, and in the lower part - coarse sand with gravel which contains one, two or three layers of mild clay and sludge of different thickness. We decompose this area into three parts according to the permeability. The section graph and plane graph are listed in Figures $\mathbf{1}$ and 2, respectively. The geological parameters are listed in Table 1, where No., CP, RWS, SY, DD and ICP denote zone number, coefficients of permeability, rate of water, specific yield, dispersion degree and infiltration coefficient of precipitation.

\begin{tabular}{|c|c|c|c|c|c|c|c|}
\hline \multirow{2}{*}{ No. } & \multicolumn{2}{|c|}{$\mathrm{CP}(\mathrm{m} / \mathrm{d})$} & \multirow{2}{*}{$\frac{\mathrm{RWS}\left(\mathrm{m}^{-1}\right)}{S_{s}}$} & \multirow{2}{*}{$\begin{array}{l}\text { SY } \\
S_{y}\end{array}$} & \multicolumn{2}{|c|}{$\mathrm{DD}(\mathrm{m})$} & \multirow{2}{*}{ ICP } \\
\hline & $K_{x x}=K_{y y}$ & $K_{z z}$ & & & $\alpha_{L}$ & $\alpha_{T}$ & \\
\hline $\mathrm{A}$ & 17 & 15 & $8.0 \times 10^{-5}$ & 0.075 & 8.3 & 0.001 & 0.3 \\
\hline B & 103 & 22 & $1.2 \times 10^{-4}$ & 0.13 & 8.3 & 0.001 & 0.3 \\
\hline $\mathrm{C}$ & 7 & 7 & $5.0 \times 10^{-5}$ & 0.04 & 0.08 & 0.0004 & 0.3 \\
\hline $\mathrm{D}$ & 63 & 17 & $1.0 \times 10^{-4}$ & 0.11 & 0.08 & 0.0004 & 0.3 \\
\hline
\end{tabular}

Let $h_{x}=20 \mathrm{~m}, h_{y}=30 \mathrm{~m}, h_{z}=1 \mathrm{~m}$. We compare our results, real values and the results of others. A represents the results of Nanjing University [30], and B represents our results. The comparison of graphs of water head and concentration are listed in Figures 3 and 4, respectively. The section graphs for water and concentration are listed in Figures 5 and 6.

Table 1. The geological parameters. 


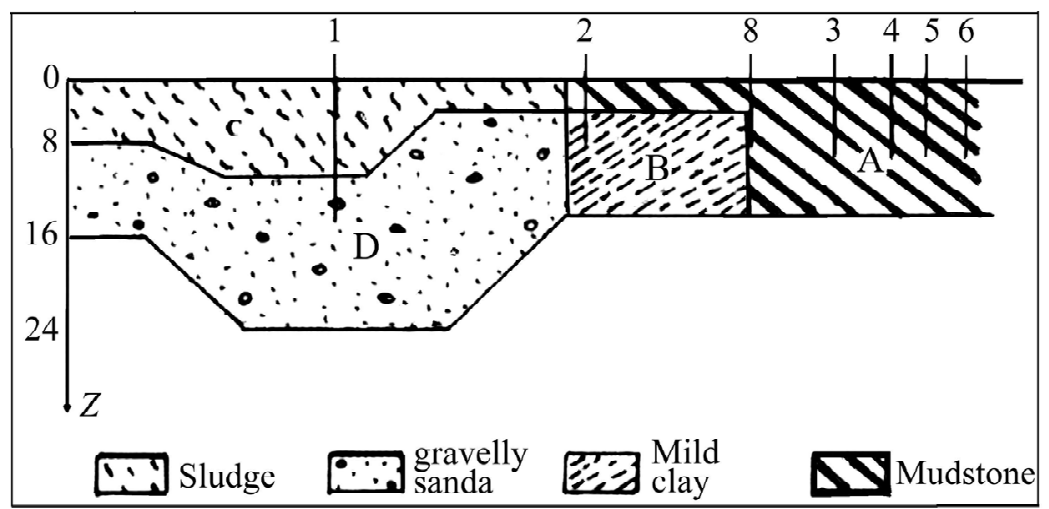

Figure 1. The section graph.

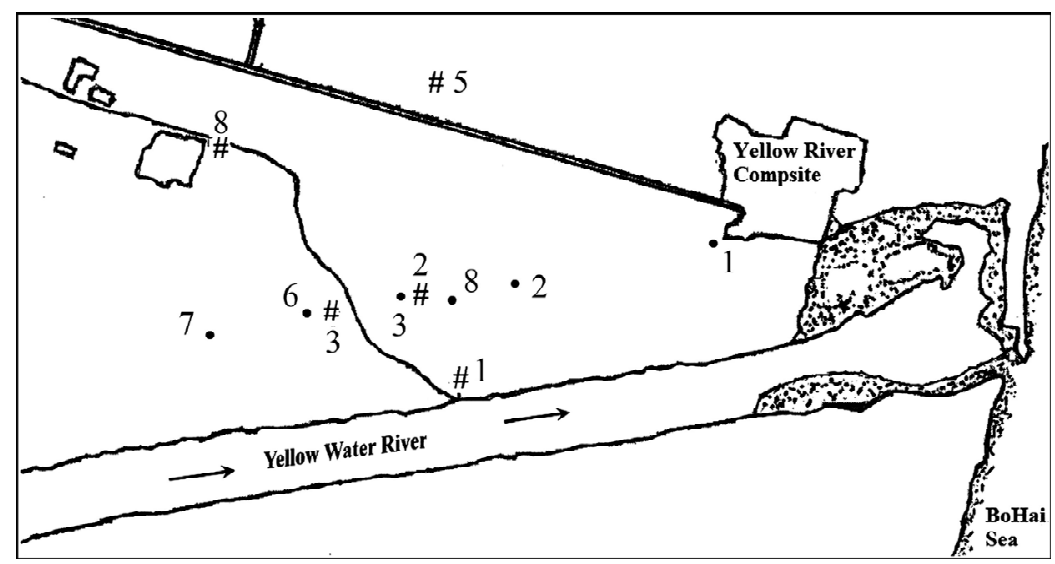

Figure 2. The plane graph.

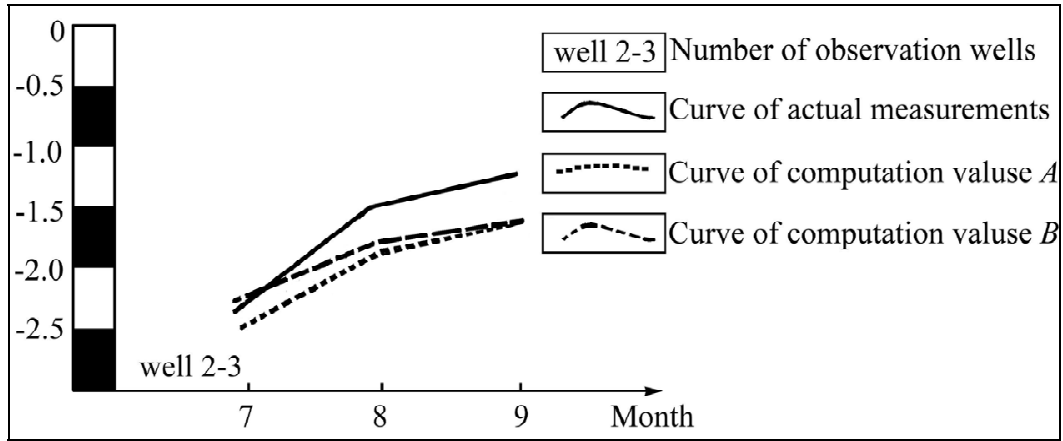

Figure 3. Curves of water level comparison.

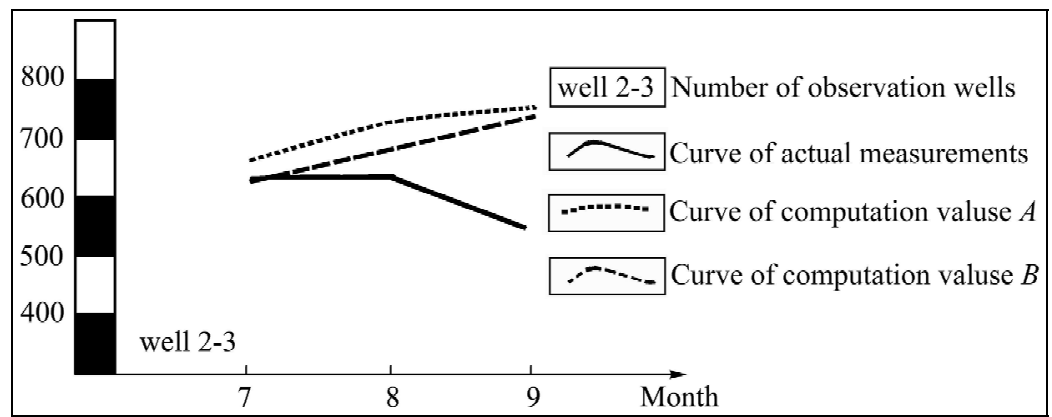

Figure 4. Curves of concentration comparison. 


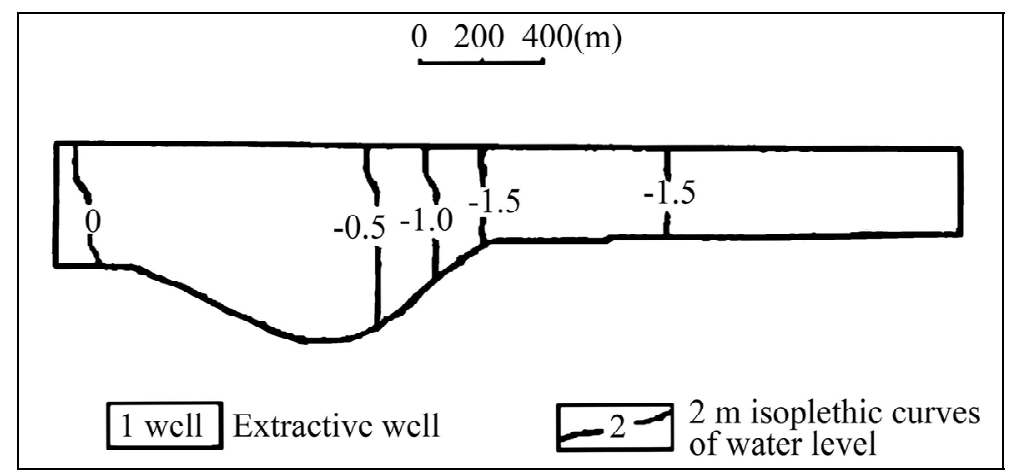

Figure 5. Section graph of water level computation in September.

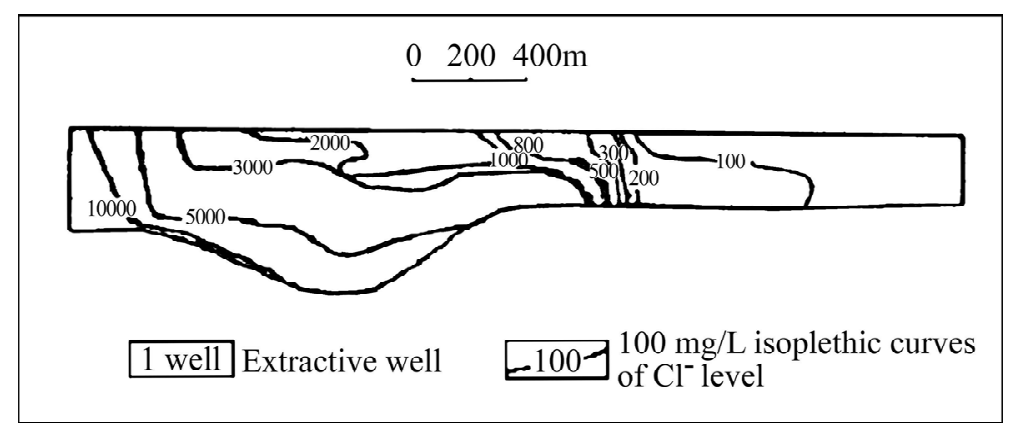

Figure 6. Section graph of $\mathrm{Cl}^{-}$concentration in September.

From the above we can see that the computation results are exact, and the algorithm given in this paper is stable and can be used as the algorithm for the simulation of large-scaled problems.

\section{Consequences of Protection Projects and Applied Modular Form of Project Adjustment}

\subsection{Consequences of Projection Projects}

The main water conservancy works against seawater intrusion include projects for water saving, Yellow River regulation, water retaining and artificial precipitation. Their ultimate goals are to increase water supplies and to decrease the extraction of underground water the production of human and animal needed, so that the descent of underground water level will be slowed down and even underground water be increased. All this is very effective. Up to now, the protection project results are mainly empirical and qualitative. We have not seen publications both in China and abroad about the real salt water and fresh water movement changes after the projects are simulated with computers. There are no publications on the quantitative and comprehensive predictions of vari- ous projects. Now we take watersaving project as an example to discuss the predictive result of the projects.

Scheme: Keep the present precipitation level. Take into consideration the effects of water-saving project on seawater intrusion. Take the average precipitation amount in many years (Refer to "Comprehensive Control Plan Against Seawater Intrusion in Laizhou Bay Area of Shandong Province"). Simulate water levels and changes of salt concentration two months after the peak period (July-August) in the following four conditions: the present pumping out, saving water $10 \%$, saving water $20 \%$ and saving water $30 \%$. Water heads and concentrations of some wells at initial time are listed in Table 2. The calculation and comparison results are listed in Tables 3 and 4. The predictive sections at water saving $20 \%$ are listed in Figures 7 and $\mathbf{8}$.

From the above we can see the consequences of water saving projects are remarkable. During raining seasons the underground water level rises again quickly. In dry seasons, its descent is slowed down. So the projects slow down the migration of salt concentration to fresh water

Table 2. The initial values of water head and concentration.

\begin{tabular}{ccc}
\hline Well No. & Water head $(\mathrm{m})$ & Salt concentration $(\mathrm{mg} / \mathrm{L})$ \\
\hline $1-2$ & -1.01 & 3667 \\
$2-2$ & -2.20 & 3000 \\
$3-2$ & -2.77 & 377 \\
$4-2$ & -3.10 & 400 \\
$5-2$ & -3.13 & 98 \\
$6-2$ & -2.87 & 100 \\
\hline
\end{tabular}


Table 3. The effects of water saving projects on water head.

\begin{tabular}{ccccccc}
\hline \multirow{2}{*}{ Saving water } & \multicolumn{7}{c}{ Well number, Water head } \\
\cline { 2 - 7 } & $1-2$ & $2-2$ & $3-2$ & $4-2$ & $5-2$ & $6-2$ \\
\hline 0 & -0.45 & -1.75 & -2.04 & -2.42 & -2.32 & -2.16 \\
$10 \%$ & -0.34 & -1.52 & -1.80 & -2.14 & -2.07 & -1.93 \\
$20 \%$ & -0.23 & -1.31 & -1.56 & -1.87 & -1.81 & -1.71 \\
$30 \%$ & -0.12 & -1.10 & -1.33 & -1.60 & -1.55 & -1.48 \\
\hline
\end{tabular}

Table 4. The effects of water saving projects on salt concentration.

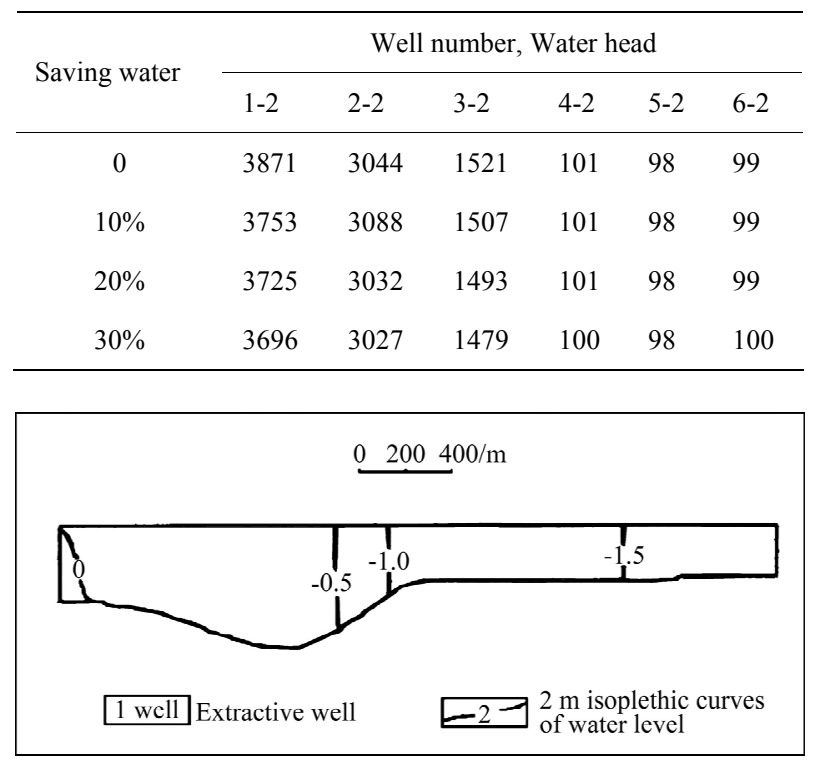

Figure 7. The water head prediction at $20 \%$ water saving in July-August.

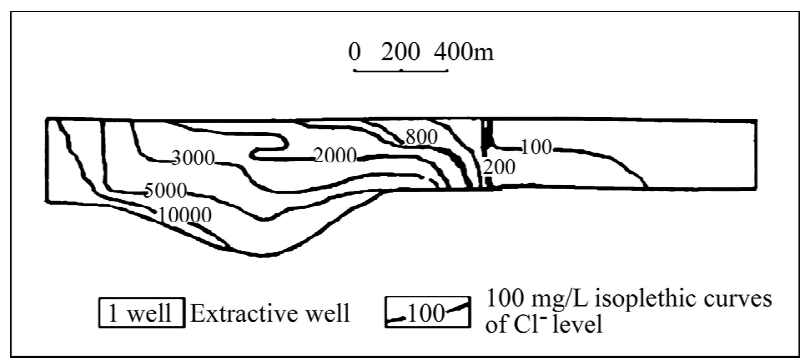

Figure 8. The salt concentration prediction at $20 \%$ water saving in July-August.

areas.

\subsection{Predicting the Consequences of Underground Dam and Tidal Barrage Projects}

Underground dams and reservoirs are built with the aim to regulation underground water and prevent seawater intrusion. Underground cut-off walls are built to stop undercurrent and seawater, since water head is low and the dams are located under the ground, so the stability and safety are high. We can say that the seepage control ability of dams is the key point. Our practice indicates the following four effects. Firstly, underground dams stop undercurrent and increase water supply, playing the role of saving and regulating water. Secondly, they raise underground water level and increase artificial precipitation supply coefficient, playing the role of retaining and supplying water. Thirdly, the upper reaches dams in seawater invaded regions retain and regulate underground water, increase the height of the fresh water heads in upper reaches, relieving the present seawater intrusion and thus playing an important role for seawater invaded regions in lower reaches. Finally, the dams in lower reaches (near the coast) prevent seawater intrusion. Tidal barrages are usually composed of two parts: the part on the ground and the underground base. Our analysis indicates that both parts are very useful. The on-the-ground part prevents seawater coming in with windstorms, while the underground part prevents seawater intrusion in common situations because of its small permeability. The advantages of tidal barrages especially obvious in of windy period.

There usually are two kinds of anti-percolator, namely: lower reaches dam in seawater invaded region, and upper reaches dam in seawater invaded region.

Lower reaches dams should be built on rivers which empty into the sea, or in other places where both salt and fresh water move freely. If a large amount of underground water is pumped out in coastal areas, water level goes down rapidly. When underground water level is lower than the average tidal level, seawater intrusion happens. This is because of the continuity between inland fresh water and seawater. Underground dams reduce greatly or completely stops the permeability of autochthonous layer. Therefore, cut-off walls can reduce the possibility of the seawater in lower reaches intruding inland. Moreover, they can retain and regulate the drainage of underground water. They stop seawater intrusion thanks to the combined actions of their own and fresh water curtain. Underground dams should be located far from the upstream of tides with the consideration of tide actions. Otherwise, tidal barrages should he built whose upper part is barrage and the lower part is underground dam.

As for the upper reaches dams in seawater intruded areas, since the intrusion has occurred, the underground walls must be built at the head of these areas with the aim to retain underground water and increase the fresh water head from upper reaches and to make seawater intrusion stable. This is also useful for inland fresh water areas far from the coast because these dams prevent the decrease of fresh water amount going into the sea, and 
thus prevent the descent of underground water level in the upper area of intruded areas. The descent of underground water level accelerates seawater going into the inner part of fresh water areas.

We predicted the effects of the dams on both upper and lower reaches on seawater intrusion. We chose the above-mentioned calculation regions. The results are shown in Table 5. Figure 9 shows the calculated concentration comparison curve. Where A is the depth of the lower reaches dam $0 \mathrm{~m}$. water level of the upper reaches is $-1.5 \mathrm{~m}$. B is the depth of lower reaches dam $2 \mathrm{~m}$. Water level of the upper reaches is $-1 \mathrm{~m}$. C is the depth of lower reaches dam $4 \mathrm{~m}$. Water level of upper reaches is $-0.5 \mathrm{~m}$. D is the depth of the lower reaches dam $6 \mathrm{~m}$. Water level of upper reaches is $0 \mathrm{~m}$.

\subsection{Applied Modular Form of Project Adjustment}

We should also apply numerical simulation to make underground water mechanics serve our goal. As for water supply, we should study how to make the limited underground water resources exert the most social and economic benefits, how to limits underground water level descent within our control and how make water supply reach the utmost. As for the protection of natural resources, we should study how to control pollutant discharge and prevent underground water being polluted, and how to keep water quality within the permission of hygienic standards. Here we propose the optimal

Table 5. $\mathrm{Cl}^{-}$concentration computation with the effect of upper reaches and lower reaches dams (after two months).

\begin{tabular}{ccccccc}
\hline \multirow{2}{*}{$\begin{array}{c}\text { Computation } \\
\text { condition }\end{array}$} & 1 & 2 & 3 & 4 & 5 & 6 \\
\cline { 2 - 7 } & 0.103 & 0.186 & 2.148 & 4.019 & 10.900 & 15.046 \\
A & 0.103 & 0.184 & 2.142 & 3.999 & 10.678 & 14.800 \\
B & 0.103 & 0.182 & 2.136 & 3.987 & 10.460 & 14.531 \\
C & 0.103 & 0.180 & 2.132 & 4.006 & 10.325 & 14.358 \\
D & & & & & & \\
\hline
\end{tabular}

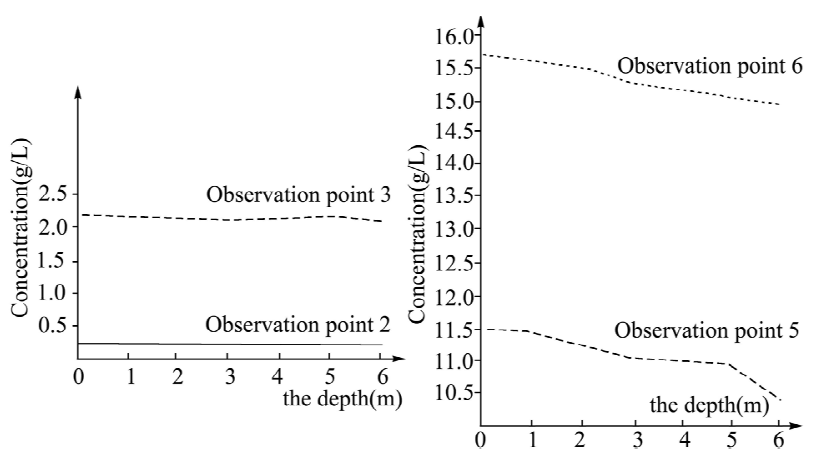

Figure 9. Curves of concentration comparison (two months). method (linear programming) and numerical method. By their combined efforts the modular form is optimized and controlled. Namely, we take underground water variables (water level, discharge, concentration and so on) in differential equations as the decision variables, use difference method change them into linear algebraic equation groups and introduce them into linear programming model as the constraint conditions.

Now we perform numerical simulation of a real project. Assume that there are two pumping wells lying in some area, whose quantities of pumping water are 4940 $\mathrm{m} / \mathrm{d}$ and $4227 \mathrm{~m} / \mathrm{d}$, respectively. Taking some observed well A near pumping wells as a new observed well and applying previous methods, we optimize and study adjusted project modes under different cases.

Let it be supposed that the maximum quantity of pumping water of each well is never more than 5000 $\mathrm{m}^{3} / \mathrm{d}$ during winter without any rain. Three cased are considered here to optimize the quantity of each well with adjusted computation. The first case is that the groundwater level of observed well doesn't decrease (Case 1). The second case is that the increase of the level is less than $0.1 \mathrm{~m}$ (Case 2). And the last case is that the increase of the level is more than $0.1 \mathrm{~m}$ (Case 3). Numerical data under three cases described above are illustrated in Table 6.

With three cases considered above, prediction for seawater intrusion problems is shown in Table 7, and the salt concentration of Case 1 is shown in Figure 10.

From the data in tables, it is easily seen that the second pumping well affects more heavily than the first one as for the level of observed wells. Thus, we can draw the following conclusions.

1) For the fixed pumping well and observed well, the

Table 6. Adjusted mode of water saving project $\left(\mathrm{m}^{3} / \mathrm{d}\right)$.

\begin{tabular}{cccc}
\hline \multirow{3}{*}{ Quantity } & \multicolumn{3}{c}{ Pumping well number } \\
\cline { 2 - 4 } & 1 & 2 & 3 \\
\hline Case 1 & 5000 & 1840 & 6840 \\
Case 2 & 5000 & 1620 & 6620 \\
Case 3 & 5000 & 2050 & 7050 \\
\hline
\end{tabular}

Table 7. Changes of salt concentration in soil under different conditions $(\mathrm{mg} / \mathrm{L})$.

\begin{tabular}{ccccccc}
\hline \multirow{2}{*}{ Water head } & \multicolumn{7}{c}{ Observation point } \\
\cline { 2 - 7 } & $1-1$ & $2-2$ & $3-2$ & $4-2$ & $5-2$ & $6-2$ \\
\hline Case 1 & 3875 & 3043 & 1494 & 101 & 98 & 100 \\
Case 2 & 3870 & 3042 & 1491 & 101 & 98 & 100 \\
Case 3 & 3924 & 3056 & 1526 & 101 & 98 & 99 \\
\hline
\end{tabular}




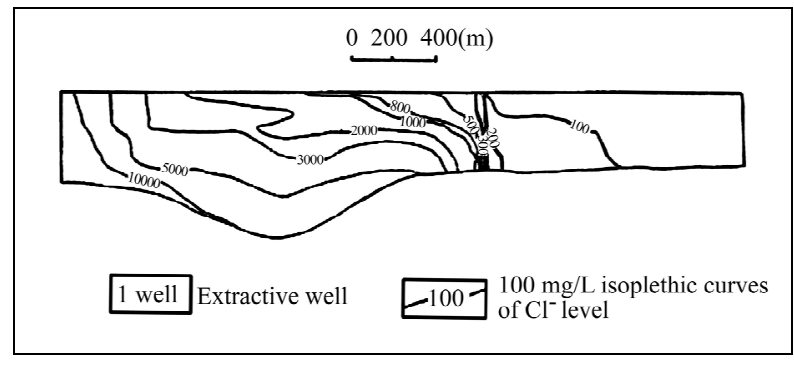

Figure 10. The salt concentration of Case 1.

first pumping well can work in the usual form while the second one should be strictly restricted by applying some water saving rules nearby.

2) Needs of the second pumping well should be firstly considered in Yellow River Diversion Project.

If the locations of pumping wells and observation points are different, the regulation modular forms are different too. With the establishment of ecological and environmental control projects, it is possible to get the timely and accurate observation data about seawater intrusion. Therefore, the established control model can do control model calculation for all projects in the entire region.

\section{Acknowledgements}

This work was supported by the Major State Basic Research Development Program of China (Grant No. G19990328), the National Tackling Key Problems Program (Grant No. 20050200064), the National Natural Sciences Foundation of China (Grant Nos. 10771124, 11101244, 10372052), the Doctorate Foundation of the State Education Commission(Grant No.20030422047), the Natural Science Foundation of Shandong Province (Grant No. ZR2009AQ012), and Independent Innovation Foundation of Shandong University (Grant No. 2010TS 031). The authors want to thank Prof. Ewing R. E and Prof. Lishang Jiang for their discussion and suggestions.

\section{REFERENCES}

[1] J. Bear, "Dynamics of Fluids in Porous Media," American Elsevier Publishing Company, Inc., Amsterdam, 1972.

[2] Y. Yuan, D. Liang and H. Rui, "The Mathematics Model of the Consequence of Predictive Seawater Intrusion and Protection Projects," In: E. Jiang, Ed., Proceeding of the 2nd Higher Mathematics Research of Shandong Province, Qingdao Ocean University Press, Qingdao, 1994, pp. 1-5.

[3] H. R. Henry, "Effects of Dispersion on Salt Water Encroachment in Coastal Aquifers," US Geological Surrey, Water Supply Paper, 1964, pp. 1613-1624.

[4] G. Segol, G. F. Pinder and W.G. Gray, "A Galerkin Finite Element Technique for Calculating the Transient Position of the Saltwater Front," Water Resources Research, Vol.
11, No. 2, 1975, pp. 343-347. doi:10.1029/WR011i002p00343

[5] P. S. Huyakorn, P. S. Anderson and J. W. Mercer, "Saltwater Intrusion in Aquifers: Development and Testing of a Three Dimensional Finite Element Model," Water Resources Research, Vol. 23, No. 2, 1987, pp. 293-312. doi:10.1029/WR023i002p00293

[6] A. D. Gupta and D. D. Yapa, "Saltwater Encroachment in an Aquifer: A Case Study," Water Resources Research, Vol. 18, No. 3, 1982, pp. 546-556. doi:10.1029/WR018i003p00546

[7] J. Douglas Jr. and T. F. Russell, "Numerical Methods for Convection-Dominated Diffusion Problems Based on Combining the Method of Characteristics with Finite Element or Finite Difference Procedures," SIAM Journal of Numerical Analysis, Vol. 19, No. 5, 1982, pp. 781-895.

[8] J. Douglas Jr., "Simulation of Miscible Displacement in Porous Media by a Modified Method of Characteristic Procedure," Lecture Notes in Mathematics 912, Numerical Analysis, Dundee, 1981.

[9] J. Douglas Jr., "Finite Difference Methods for Two-Phase Incompressible Flow in Porous Media," SIAM Journal of Numerical Analysis, Vol. 20, No. 4, 1983, pp. 681-696. doi: $10.1137 / 0720046$

[10] R. E. Ewing, T. F. Russell and M. F. Wheeler, "Convergence Analysis of an Approximation of Miscible Displacement in Porous Media by Mixed Finite Elements and a Modified Method of Characteristics," Computer Methods in Applied Mechanics and Engineering, Vol. 47, No. 1-2, 1984, pp. 73-92. doi:10.1016/0045-7825(84)90048-3

[11] A. Bermudez, M. R. Nogueriras and C. Vazuez, "Numerical Analysis of Convection-Diffusion-Reaction Problems with Higher Order Characteristics/Finite Elements. Part I: Time Discretization," SIAM Journal of Numerical Analysis, Vol. 44, No. 5, 2006, pp. 1829-1853. doi:10.1137/040612014

[12] A. Bermudez, M. R. Nogueriras and C. Vazuez, "Numerical Analysis of Convection-Diffusion-Reaction Problems with Higher Order Characteristics/Finite Elements. Part II: Fully Discretized Scheme and Quadrature Formulas," SIAM Journal of Numerical Analysis, Vol. 44, No. 5, 2006, pp. 1854-1876. doi:10.1137/040615109

[13] J. Douglas Jr. and J. E. Roberts, "Numerical Method for a Model for Compressible Miscible Displacement in Porous Media," Mathematics of Computation, Vol. 4, No. 164, 1983, pp. 441-459.

[14] Y. Yuan, "Time Stepping along Characteristics for the Finite Element Approximation of Compressible Miscible Displacement in Porous Media," Mathematica Numerica Sinica, Vol. 14, No. 4, 1992, pp. 385-400.

[15] Y. Yuan, "Finite Difference Methods for a Compressible Miscible Displacement Problem in Porous Media," Mathematica Numerica Sinica, Vol. 15, No. 1, 1993, pp. 16-28.

[16] R. E. Ewing, "The Mathematics of Reservoir Simulation," Society for Industrial and Applied Mathematics, Philadelphia, 1983. doi:10.1137/1.9781611971071

[17] J. Douglas Jr. and Y. Yuan, "Numerical Simulation of 
Immiscible Flow in Porous Media Based on Combining the Method of Characteristics with Mixed Finite Element Procedure," In: M. F. Wheeler, Ed., Numerical Simulation in Oil Recovery, Spring-Verlag, Minnesota, 1986, pp. 119-131.

[18] Y. Yuan, "Characteristic Finite Difference Methods for Moving Boundary Value Problem of Numerical Simulation of Oil Deposit," Science in China, Series A, Vol. 37, No. 12, 1994, pp. 1412-1453.

[19] Y. Yuan, "The Characteristic Mixed Finite Element Method and Analysis for Three-Dimensional Moving Boundary Value Problem," Science in China, Series A, Vol. 39, No. 3, 1996, pp. 276-288.

[20] Y. Yuan, "Finite Difference Method and Analysis for Three-Dimensional Semiconductor Device of Heat Conduction," Science in China, Series A, Vol. 39, No. 11, 1999, pp. 1140-1151.

[21] O. Axelsson and I. A. Gustafasson, "Modified Upwind Scheme for Convective Transport Equations and the Use of a Conjugate Gradient Method for the Solution of Non-Symmetric Systems of Equations," IMA Journal of Applied Mathematics, Vol. 23, No. 3, 1979, pp. 321-337.

[22] R. E. Ewing, R. D Lazarov and A. T. Vassilevski, "Finite Difference Scheme for Parabolic Problems on Composite Grids with Refinement in Time and Space," SIAM Journal on Numerical Analysis, Vol. 31, No. 6, 1994, pp. 1605-1622. doi: $10.1137 / 0731083$

[23] R. D. Lazarov, I. D. Mishev and P. S. Vassilevski, "Finite Volume Method for Convection-Diffusion Problems,"
SIAM Journal on Numerical Analysis, Vol. 33, No. 1, 1996, pp. 31-55. doi:10.1137/0733003

[24] D. W. Peaceman, "Fundamentals of Numerical Reservoir Simulation," Elsevier, Amsterdam, 1980.

[25] G. I. Marchuk, "Splitting and Alternating Direction Method," In: P. G. Ciarlet and J. L. Lions, Eds., Handbook of Numerical Analysis, Elsevior Science Publishers BV, Paris, 1990, pp. 197-460. doi:10.1016/S1570-8659(05)80035-3

[26] J. Douglas Jr. and J. E. Gunn, "Two Order Correct Difference Analogues for the Equation of Multidimensional Heat Flow," Mathematics of Computation, Vol. 17, No. 81, 1963, pp. 71-80. doi:10.1090/S0025-5718-1963-0149676-2

[27] J. Douglas Jr. and J. E. Gunn, “A General Formulation of Alternation Methods. Part 1. Parabolic and Hyperbolic Problems," Numerische Mathematik, Vol. 9, No. 5, 1964, pp. 428-453.

[28] R. E. Ewing, "Mathematical Modeling and Simulation for Multiphase Flow in Porous Media. In Numerical Treatment of Multiphase Flow in Porous Media," Lecture Notes in Physics, Vol. 552, 2000, pp. 43-57.

[29] A. A. Samarski and B. B. Andreev, "Finite Difference Methods for Elliptic Equation," Science Press, Beijing, 1984.

[30] Y. Xue and C. Xie, "Study on Joint-Surface of Saltwater and Freshwater in Seawater Intrusion Problem," Nanjing University Press, Nanjing, 1991, pp. 43-94. 